\title{
Prospektive und prozessbezogene Kompetenzanalyse im Digitalisierungskontext - Gestaltung und Erprobung eines Vorgehens
}

\author{
Sarah Depenbusch ${ }^{1}$ Elena Bender ${ }^{1} \cdot$ Niclas Schaper $^{1}$ \\ Angenommen: 23. September 2021 / Online publiziert: 13. Oktober 2021 \\ (c) Der/die Autor(en) 2021
}

\section{Zusammenfassung}

Im Zuge der Digitalisierung von Arbeits- und Geschäftsprozessen ist es wichtig, Kompetenzanforderungen zukunftsbezogen und konkret zu ermitteln, um Entwicklungsbedarfe frühzeitig zu erkennen. Dieser Beitrag untersucht daher, wie zukünftige Kompetenzen zugleich prospektiv und prozessbezogen erfasst werden können. Dazu wird ein Vorgehen erprobt, welches verschiedene Verfahren der Anforderungsanalyse integriert. In der gegenwartsbezogenen Analysephase werden zunächst die Kompetenzen ermittelt, die für den Prozess vor dessen digitaler Veränderung relevant sind, und in gegenwarts- und prozessbezogenen Kompetenzprofilen zusammengefasst. In der zukunftsbezogenen Analysephase werden anschließend die für den veränderten, digitalisierten Prozess erforderlichen Kompetenzen hergeleitet und in zukunfts- und prozessbezogenen Kompetenzprofilen abgebildet. Die Kompetenzerhebung erfolgt in beiden Analysephasen mittels Experteninterviews, in denen dieselben Befragungsinstrumente eingesetzt werden. Durch einen Vergleich der gegenwarts- und zukunftsbezogenen Kompetenzprofile lassen sich schließlich zukünftige Kompetenzentwicklungsbedarfe der Mitarbeitenden prozessbezogen und prospektiv ableiten.

Praktische Relevanz: Das Vorgehen ermöglicht Unternehmen die prospektive und prozessbezogene Erfassung zukünftig relevanter Kompetenzen und Entwicklungsbedarfe sowie die Gestaltung individueller und prozessorientierter Kompetenzentwicklungsmaßnahmen, insbesondere im Digitalisierungskontext. Die prospektive Ableitung des zukünftigen Entwicklungsbedarfs ist wichtig, um technologisch mit dem digitalen Wandel Schritt zu halten und die Mitarbeitenden dabei proaktiv mit einzubeziehen.

Schlüsselwörter Digitalisierung $\cdot$ Kompetenzen $\cdot$ Anforderungsanalyse $\cdot$ Prospektive und prozessbezogene Kompetenzanalyse $\cdot$ Kompetenzprofil

\section{Prospective and process-related competence analysis in the digitization context-Design and testing of an approach}

\begin{abstract}
In the course of the digitization of work and business processes, it is important to record competences future-oriented and concretely in order to prematurely identify development needs. This article therefore examines how future competences can be recorded both prospectively and process-related. For this purpose, an approach is being tested that integrates various methods of requirements analysis. In the present-related analysis phase, the competences relevant for the current work process are recorded and then transferred into present- and process-related competence profiles. In the future-related analysis phase, the competences relevant for the changed, digitized process are recorded and summarized in future- and process-related competence profiles. In both analysis phases, the current or future competences are assessed in expert interviews, utilizing the same survey instruments. By comparing the current- and future-related competence profiles, future needs for competence development are derived and analysed in a prospective and process-related manner.
\end{abstract}

Sarah Depenbusch, M.Sc.

Institut für Humanwissenschaften - Fach Psychologie, Universität Paderborn, Warburger Str. 100, 33098 Paderborn,

sarah.depenbusch@uni-paderborn.de 
Practical Relevance: The presented approach enables companies to prospectively record future development needs in relation to a specific work process and to design individual and process-oriented measures for competence development, especially in the context of digitization. The prospective derivation of future development needs is important to keep pace with the digital change and to proactively involve employees during this change.

Keywords Digitization - Competences · Requirements analysis · Prospective and process-related competence analysis · Competence profile

\section{Hintergrund und Relevanz}

Durch den digitalen Wandel verändern sich betriebliche Prozesse zu flexiblen und vernetzten Wertschöpfungsketten (Hirsch-Kreinsen 2017; Leyh und Bley 2016; Urbach et al. 2019). In der heutigen Zeit unterstützen neue Technologien Mitarbeitende nicht nur bei der Ausführung manueller und kognitiver Routinetätigkeiten (Dengler und Matthes 2015; Rothe et al. 2019), sondern auch bei der Verarbeitung vielfältiger Informationsflüsse (Urbach und Ahlemann 2019) und der Durchführung komplexer Arbeitstätigkeiten (Coovert und Thompson 2014; Paruzel et al. 2020). Die Nutzung digitaler Technologien wirkt sich jedoch nicht nur auf die Arbeitsinhalte und -organisation aus (Carstensen 2015; Rothe et al. 2019), sondern verändert ebenfalls die damit verbundenen Arbeitsanforderungen an die Mitarbeitenden (Enke et al. 2018; Harteis 2018; van Laar et al. 2020). Durch den Rückgang repetitiver und standardisierter Tätigkeiten in digitalisierten Arbeitssystemen erhalten Mitarbeitende oftmals höhere Verantwortungs- und Entscheidungsbefugnisse (Galiläer 2006). In digitalen Arbeitsumgebungen gewinnen daher u.a. die Kommunikations- und Teamfähigkeit, die Flexibilität und Anpassungsfähigkeit (Finegold und Notabartolo 2010), die Kreativität und Problemlösefähigkeit (Kinkel et al. 2017) sowie die Belastbarkeit (Galiläer 2006) und Verantwortungsfähigkeit der Mitarbeitenden (Zinke 2019) an Bedeutung. Zudem werden insbesondere digitale Kompetenzen wichtig, welche sich u. a. auf Fähigkeiten zur Daten- und Informationsverarbeitung oder die Kommunikation und Zusammenarbeit mittels digitaler Technologien beziehen (van Laar et al. 2017; Vuorikari et al. 2016).

Um veränderte Anforderungen an die Mitarbeitenden zu identifizieren, wurden bereits erste Ansätze zur prospektiven Anforderungsanalyse entwickelt, welche bestehende arbeits- und anforderungsanalytische Verfahren zukunftsbezogen einsetzen (z. B. Kato-Beiderwieden et al. 2021; Koch 2010; Koch et al. 2012). Zukünftige Anforderungen und Kompetenzen sollten jedoch nicht nur prospektiv, sondern auch möglichst konkret, d.h. in Bezug auf den digitalisierten bzw. noch zu digitalisierenden Prozess, erfasst werden. Bislang werden prospektive und prozessbezogene Analyseverfahren jedoch häufig unabhängig voneinander betrachtet bzw. deren Integration eher generisch beschrieben. Der vorliegende Beitrag widmet sich dieser Forschungslücke und erprobt ein Vorgehen, welches den prospektiven und prozessbezogenen Analysefokus miteinander kombiniert und in einer integrierten Form berücksichtigt. Hierbei werden verschiedene Verfahren der Anforderungsanalyse (z. B. Critical Incident Technique (CIT), Flanagan 1954; Work Design Questionnaire (WDQ), Stegmann et al. 2010) integriert und schließlich zukunfts- und prozessbezogen eingesetzt. Das resultierende Vorgehen untergliedert sich in eine gegenwarts- und eine zukunftsbezogene Analysephase. In der gegenwartsbezogenen Analysephase werden als Vergleichsbasis die für den bestehenden Prozess relevanten Kompetenzen erfasst und in prozessbezogenen Kompetenzprofilen abgebildet. Dazu werden zunächst ein generisches Kompetenzmodell und darauf aufbauend entsprechende tätigkeitsbezogene Kompetenzmodelle entwickelt. Indem die in den tätigkeitsbezogenen Kompetenzmodellen abgebildeten Kompetenzfacetten den einzelnen Prozessschritten der jeweiligen Tätigkeitsbereiche zugeordnet werden, entstehen schließlich die gegenwarts- und prozessbezogenen Kompetenzprofile. In der zukunftsbezogenen Analysephase werden die für den veränderten, digitalisierten Prozess erforderlichen Kompetenzen ermittelt und auf die gleiche Art und Weise in prozessbezogene Kompetenzprofile überführt. Beide Analysephasen erfolgen unter Einsatz derselben Befragungs- und Begleitinstrumente, welche in diesem Beitrag vorgestellt werden. Darüber hinaus wird verdeutlicht, wie zukünftige Kompetenzentwicklungsbedarfe prospektiv und prozessbezogen analysiert werden können.

\section{Theoretische Grundlagen}

Im Folgenden werden zunächst der Anforderungs- und der Kompetenzbegriff definiert. Anschließend werden Ansätze der Anforderungsanalyse sowie der Kompetenzanalyse bzw. des Kompetenzmanagements präsentiert, welche als theoretische Grundlagen zur Gestaltung der prospektiven und prozessbezogenen Kompetenzanalyse herangezogen wurden.

\subsection{Anforderungen und Kompetenzen}

Anforderungen beschreiben die zur Erfüllung einer Tätigkeit oder Position erforderlichen Personenmerkmale 
(Schaper 2019). Dabei kann es sich um fachliches Wissen (z.B. Produktkenntnisse), kommunikative und kooperative Fähigkeiten (z.B. Teamfähigkeit) oder bestimmte Persönlichkeitseigenschaften (z.B. Zuverlässigkeit) handeln (Blickle 2019). Kompetenzen charakterisieren die Fähigkeit von Menschen, in komplexen Situationen effektiv zu handeln, indem geforderte Personenmerkmale zielführend eingesetzt werden (Eck et al. 2007; Krumm et al. 2012). Deshalb werden Kompetenzen hier auch als spezifische Anforderungsbeschreibungen aufgefasst. Der im Kontext des beruflichen Handelns verwendete Begriff der beruflichen Handlungskompetenz beruht ebenfalls auf einem solchen ganzheitlichen Verständnis und fokussiert daher neben fachlichen und methodischen auch soziale, motivationale und emotionale Faktoren des Arbeitshandelns (Sonntag und Schaper 1999; Sonntag und SchmidtRathjens 2005). Das wohl am meisten verbreitete Strukturierungsschema von Kompetenzen, und somit auch der beruflichen Handlungskompetenz, ist die Untergliederung in die Bereiche der Fach-, Methoden-, Sozial- und Selbstkompetenz (Sonntag und Schaper 2016). Fachkompetenzen beziehen sich auf spezifische Kenntnisse und Fertigkeiten, die für die Bewältigung beruflicher Aufgaben erforderlich sind (z.B. Kenntnisse und Fertigkeiten zur Prüfung von Produktparametern). Methodenkompetenzen beinhalten situationsübergreifend einsetzbare (meta-)kognitive Fähigkeiten (z.B. Problemlösefähigkeit), die zur selbstständigen Ausführung von komplexen Arbeitsaufgaben erforderlich sind (Schaper 2019). Sozialkompetenzen umfassen Fähigkeiten zum zielorientierten Handeln in sozialen Interaktionssituationen (z.B. Kommunikationsfähigkeit) (Kauffeld und Grote 2019). Selbstkompetenzen beziehen sich zum einen auf persönlichkeitsbezogene Dispositionen wie Einstellungen und Motive, die das Arbeitshandeln beeinflussen (z.B. Verantwortungsübernahme) und zum anderen auf Fähigkeiten zur eigenständigen Steuerung des beruflichen Handelns (z. B. Lernfähigkeit) (Schaper 2019). Zusätzlich zu diesen in der Literatur breit beschriebenen Kompetenzbereichen gewinnt in der aktuellen Situation das Konzept der digitalen Kompetenz an Bedeutung (Ferrari 2012, 2013). Gemäß dem europäischen Referenzrahmen für digitale Kompetenzen (DigComp 2.1, Carretero et al. 2017) setzt sich die digitale Kompetenz aus fünf Kernbereichen zusammen. Diese umfassen die Informations- und Datenkompetenz (z. B. Recherche und Evaluation digitaler Daten), die Kommunikation und Zusammenarbeit mittels digitaler Technologien (z.B. Nutzung von Informationsund Kommunikationstechnologien), das Erzeugen digitaler Inhalte (z.B. Erstellen von Computereintragungen), die Gewährleistung der IT-Sicherheit (z.B. Datenschutz) und das Problemlösen mittels digitaler Technologien (z. B. Lösen technischer Probleme) (Carretero et al. 2017). Da sich die Kernbereiche der digitalen Kompetenz größtenteils in die vier Kompetenzbereiche der Fach-, Methoden-, Sozialund Selbstkompetenz einordnen lassen, handelt es sich bei der digitalen Kompetenz um eine Querschnittskompetenz (Blumberg und Kauffeld 2021).

\subsection{Traditionelle Formen der Anforderungsanalyse}

Berufliche Anforderungen und Kompetenzen lassen sich sowohl durch traditionelle Anforderungsanalysen als auch durch Ansätze des betrieblichen Kompetenzmanagements ableiten (Höft und Goerke 2014). Im Rahmen traditioneller Anforderungsanalysen werden die für eine Position oder Tätigkeit erforderlichen Anforderungen ermittelt und in Kompetenzprofilen zusammengefasst (Lorenz und Rohrschneider 2015). Um Anforderungen umfassend $\mathrm{zu}$ erheben, sollten verschiedene Methoden der Anforderungsanalyse kombiniert werden (Blickle 2019; Schuler 2002). Die Literatur unterscheidet zwischen drei methodischen Herangehensweisen: Bei der erfahrungsgeleitet-intuitiven Methode führen Personen mit langjähriger Erfahrung bzgl. bestimmter Tätigkeiten oder Berufe (z. B. Arbeitsanalytiker) die Anforderungsanalyse durch (Blickle 2019). Ein in diesem Rahmen häufig angewendetes Verfahren ist die „Critical Incident Technique“ (CIT, Flanagan 1954; Krüger et al. 2015; Lozo et al. 2015). Hierbei sollen durch die Arbeitspersonen konkrete Verhaltensweisen beschrieben werden, die in einer herausfordernden Arbeitssituation (kritisches Ereignis) zu einer besonders effektiven oder ineffektiven Aufgabenerfüllung geführt haben (Flanagan 1954; Hamborg und Schaper 2018). Neben den effektiven Verhaltensweisen werden dabei ebenfalls der Hintergrund und die Konsequenzen des kritischen Ereignisses betrachtet (Eck und Rietiker 2010). Im Rahmen der personenbezogen-empirischen Methode werden Anforderungen anhand von statistischen Zusammenhängen zwischen bestimmten Personenmerkmalen und verschiedenen Kriterien der Leistungsbeurteilung ermittelt. Dazu wird bspw. auf Metaanalysen zurückgegriffen, die entsprechende einschlägige Studien zusammenfassen (Blickle 2019). Bei der arbeitsplatzanalytisch-empirischen Methode beruht die Anforderungsanalyse auf einer zuvor durchgeführten Arbeitsanalyse (Blickle 2019). Ziel der psychologischen Arbeitsanalyse ist die Analyse und Bewertung von Arbeitstätigkeiten sowie ihrer Bedingungen und Auswirkungen auf das Individuum. Dabei werden systematisch Informationen $\mathrm{zu}$ Arbeitsaufgaben, -mitteln und -produkten sowie der Arbeitsumgebung gesammelt und analysiert (Schaper 2019). Basierend auf diesen Informationen werden im Rahmen der arbeitsplatzanalytisch-empirischen Methode anschließend die Personenmerkmale abgeleitet, welche die Arbeitsperson zur erfolgreichen Tätigkeitsbewältigung benötigt (Eckhardt und Schuler 1992; Schuler 2014; Wienkamp 2020). Der ,Work Design Question- 
naire“ (WDQ) von Morgeson und Humphrey (2006) (dt. Übersetzung durch Stegmann et al. 2010) ist ein häufig eingesetztes arbeitsanalytisches Verfahren (Schaper 2019), welches eine detaillierte Erfassung der Merkmale von Arbeitstätigkeiten ermöglicht (Lozo et al. 2015; Stegmann et al. 2010). Hierbei werden aus Sicht der Mitarbeitenden neben Aufgabenmerkmalen (z.B. Aufgabenvielfalt) auch Wissensmerkmale (z.B. Informationsverarbeitung), soziale Merkmale (z.B. Unterstützung durch KollegInnen) und Kontextmerkmale (z. B. Ergonomie) identifiziert (Stegmann et al. 2010). Dadurch lassen sich die mit einer Arbeitstätigkeit verbundenen Anforderungen auf einer globalen Ebene herleiten und Verbesserungspotentiale hinsichtlich der Arbeitsgestaltung ermitteln. Durch eine Optimierung der Arbeitsgestaltungsmerkmale ist es möglich, lernförderliche Arbeitsumgebungen zu implementieren und so die Kompetenzentwicklung der Mitarbeitenden zu unterstützen (Schaper 2019).

\subsection{Kompetenzanalyse und -management}

Im Rahmen des Kompetenzmanagements werden Maßnahmen der Personalgewinnung, -entwicklung und -planung unter Zuhilfenahme betrieblicher Kompetenzmodelle durchgeführt (Grote et al. 2006; Sonntag und Stegmaier 2005). Kompetenzmanagementansätze sind im Gegensatz zu traditionellen Anforderungsanalysen auf die strategischen Ziele einer Organisation ausgerichtet (Höft und Goerke 2014; Sauter und Staudt 2016). Dennoch werden beide Ansätze häufig miteinander verknüpft: Maßnahmen des Kompetenzmanagements beruhen nicht selten auf vorangegangenen Anforderungsanalysen (Eck und Rietiker 2010). So werden bspw. die im Rahmen des strategischen Kompetenzmanagements eingesetzten Kompetenzmodelle auf Grundlage der im Rahmen von Anforderungsanalysen entwickelten Anforderungsprofile konzipiert (z.B. Competence Management Tool (CMT), Decius und Schaper 2017).

Die im Kompetenzmanagement eingesetzten Kompetenzmodelle sind zumeist organisationsspezifisch konzipiert und fassen alle Kompetenzen zusammen, die zur effektiven Leistungserbringung in einer Organisation erforderlich sind (Campion et al. 2011; Kauffeld und Grote 2019). Darüber hinaus gibt es allgemeine bzw. generische Kompetenzmodelle, die über einzelne Unternehmen hinaus bedeutsam sind (Gessler und Sebe-Opfermann 2016; Krumm et al. 2012). Der Kompetenzatlas (Heyse und Erpenbeck 2009) ist bspw. ein bekanntes generisches Kompetenzmodell, das viele Unternehmen für ihr Kompetenzmanagement nutzen (Gessler und Sebe-Opfermann 2016). Der Kompetenzatlas umfasst die übergeordneten Bereiche der Fach- und Methodenkompetenz, der Aktivitäts- und Handlungskompetenz, der sozial-kommunikativen Kompetenz und der personalen Kompetenz. Diese vier Grundkompetenzen untergliedern sich in 64 Teilkompetenzen, welche gleichzeitig bestimmten Kompetenzkombinationen zugeordnet werden können (Heyse und Erpenbeck 2009). Da es sich bei den meisten Kompetenzmodellen jedoch um reine Auflistungen relevanter Kompetenzen handelt und ihre hierarchischen Beziehungen bzw. Zusammenhänge nicht beschrieben werden, verwendet die Literatur auch oftmals den Begriff Kompetenzprofil (Krumm et al. 2012). In diesem Beitrag wird für eine differenzierte inhaltliche Darstellung jedoch zwischen beiden Begriffen unterschieden: Während Kompetenzmodelle alle für ein gesamtes Unternehmen oder einen bestimmten Tätigkeitsbereich relevanten Kompetenzen abbilden, sind Kompetenzprofile spezifischer konzipiert und führen die zur Ausführung einer bestimmten Tätigkeit notwendigen Soll-Kompetenzgrade sowie die Ist-Kompetenzgrade der Mitarbeitenden auf. Kompetenzprofile werden im Rahmen der Kompetenzanalyse genutzt, um die Kompetenzen bestimmter Arbeitspersonen zu erfassen und zu bewerten, indem die im Kompetenzprofil aufgeführten Soll-Kompetenzen mit den Ist-Kompetenzen der jeweiligen Arbeitsperson verglichen werden (Gessler und SebeOpfermann 2016; Noll 2010). Dazu werden die Ausprägungen der Soll- und Ist-Kompetenzen zuvor anhand eines Einstufungsschlüssels bestimmt (Noll 2010). Eine weitere Möglichkeit der Kompetenzmessung besteht darin, die in den Kompetenzmodellen aufgeführten Kompetenzen in einen Fragebogen zu überführen (Krumm et al. 2012). Ein weit verbreiteter Fragebogen zur Einschätzung der beruflichen Handlungskompetenz ist das Kompetenz-ReflexionsInventar (KRI, Kauffeld 2010). Das KRI untergliedert sich in die vier Bereiche der beruflichen Handlungskompetenz, wobei die jeweiligen Kompetenzdimensionen anhand einer elfstufigen Skala entweder durch die Mitarbeitenden selbst oder ihre Führungskraft eingeschätzt werden (Kauffeld 2010).

\subsection{Prospektive und prozessbezogene Anforderungs- und Kompetenzanalyse}

Da sich die Arbeitstätigkeiten sowie die damit verbundenen Anforderungen und Kompetenzen in der heutigen Zeit stetig verändern (Harteis 2018), berücksichtigen neuere Verfahren der Anforderungsanalyse bereits eine zukunftsbezogene Perspektive (Höft und Goerke 2014). Im Digitalisierungskontext ist es jedoch nicht nur wichtig, Anforderungen und Kompetenzen zukunftsbezogen, sondern auch möglichst konkret zu erfassen. Dies lässt sich insbesondere durch ein prozessorientiertes Vorgehen realisieren, wobei die für einen bestimmten Prozess oder einzelne Prozessschritte erforderlichen Kompetenzen ermittelt und in prozessbezogenen Kompetenzmodellen abgebildet werden (z.B. Hasenau et al. 2013; Hegmanns et al. 2019). Um 
den aktuellen Forschungsstand hinsichtlich prozessbezogener und prospektiver Verfahren der Kompetenz- und Anforderungsanalyse zu erfassen, erfolgte eine an das Vorgehen von Okoli (2015) angelehnte Literaturrecherche. Vor Durchführung der eigentlichen Recherche wurden zunächst die zu verwendenden Datenbanken, Suchterme sowie die im Screening-Prozess zu fokussierenden Relevanzkriterien (z.B. prospektiver Analysefokus) festgelegt. Die auf Basis dieser Kriterien durchgeführte Literaturrecherche erfolgte in den relevanten Datenbanken der Psychologie und Wirtschaftswissenschaften (z. B. Science Direct, Springer Link, WISO) und wurde durch eine Vorwärts- und Rückwärtssuche ergänzt (vom Brocke et al. 2009). Anschließend wurde der Inhalt der identifizierten Literatur erfasst und in Bezug auf bestimmte Qualitätskriterien (z.B. Einsatz qualitativer Methoden) bewertet. Die verbleibende Literatur wurde abschließend zur weiteren Bestimmung der konstatierten Forschungslücke (Gestaltung eines Vorgehens zur prospektiven und prozessbezogenen Kompetenzanalyse und seine konkrete Umsetzung und Erprobung im Rahmen spezifischer Anwendungsfälle) herangezogen.

Aus der Literaturrecherche ging hervor, dass der zukunftsbezogene Analysefokus immer wichtiger wird und mehrere Beiträge bereits Methoden zur prospektiven Kompetenzanalyse präsentieren (z.B. Lebrenz 2020; Vernim et al. 2019). Insbesondere die ,Task-Analysis-Tools“ (TAToo, Koch und Westhoff 2012) stellen eine wichtige Methodik zur prospektiven Erfassung zukünftiger Anforderungen dar. Die TAToo setzen bestehende Verfahren der Anforderungsanalyse prospektiv ein, um neben den aktuellen auch die zukünftigen Anforderungen einer Zielposition oder -tätigkeit zu erfassen (Höft und Goerke 2014; Koch 2010). Dabei wird u.a. die Critical Incident Technique (CIT, Flanagan 1954) zukunftsbezogen eingesetzt: Anhand von Beschreibungen der Befragten zu zukünftig erfolgsentscheidenden Arbeitssituationen können zukünftige Anforderungen prospektiv abgeleitet und in entsprechenden Anforderungsprofilen zusammengefasst werden (Koch 2010). Die in den TAToo vorgenommene Kombination des gegenwarts- und zukunftsbezogenen Analysefokus sowie die Integration bestehender Verfahren der Anforderungsanalyse bilden auch die Grundlage für das in diesem Beitrag beschriebene Vorgehen. Kato-Beiderwieden et al. (2021) greifen die Methodik der TAToo ebenfalls auf und kombinieren diese mit weiteren validierten Methoden zur Kompetenzerfassung, wie experimentellen VignettenMethoden und Arbeitsplatzbegehungen.

Weiterhin wurde im Rahmen der Recherche deutlich, dass bereits einige Beiträge Methoden zur prozessbezogenen Anforderungs- und Kompetenzanalyse präsentieren (z. B. Hasenau et al. 2013; Hegmanns et al. 2019; Sprafke et al. 2019). Hasenau et al. (2013) entwickeln bspw. ein Vorgehen zur Erstellung prozessbezogener Kompetenzmatrizen und beschreiben, wie in Bezug auf einzelne Prozessschritte ein Abgleich der Ist-Kompetenzen mit den jeweils geforderten Soll-Kompetenzen durchgeführt werden kann, um bestehende Kompetenzentwicklungsbedarfe der Mitarbeitenden zu identifizieren. Während Hasenau et al. (2013) den Fokus primär auf die gegenwartsbezogene Kompetenzerfassung legen, entwickeln Hegmanns et al. (2019) ein Kompetenzmanagement-Assistenzsystem, welches eine zukunftsgerichtete und prozessorientierte Kompetenzdiagnostik ermöglicht. Anhand eines prozessorientierten Kompetenzkatalogs, welcher sowohl jetzige als auch zukünftige Kompetenzanforderungen beinhaltet, können Kompetenzlücken frühzeitig identifiziert und entsprechende Kompetenzentwicklungsmaßnahmen umgesetzt werden (Hegmanns et al. 2019). Da prospektive und prozessbezogene Analyseverfahren dennoch häufig unabhängig voneinander betrachtet werden, bzw. deren Integration oftmals auf einer generischen Ebene beschrieben wird, präsentiert und erprobt der vorliegende Beitrag ein integratives Vorgehen zur prospektiven und prozessbezogenen Kompetenzanalyse in seiner konkreten Umsetzung und beschreibt die dabei eingesetzte Methodik sowie die daraus hervorgehenden Kompetenzprofile im Rahmen von praktischen Anwendungsfällen.

\section{Gegenwartsbezogene Analysephase}

Das Vorgehen zur prospektiven und prozessbezogenen Kompetenzanalyse untergliedert sich in eine gegenwartsund eine zukunftsbezogene Analysephase. Das Ziel der gegenwartsbezogenen Analysephase ist die Entwicklung von gegenwarts- und prozessbezogenen Kompetenzprofilen, welche die für einen bestehenden Arbeits- bzw. Geschäftsprozess notwendigen Kompetenzen abbilden. Die gegenwarts- und prozessbezogenen Kompetenzprofile dienen in der darauffolgenden zukunftsbezogenen Analysephase als Vergleichsbasis, um zukünftige Entwicklungsbedarfe abzuleiten.

\subsection{Methode}

Zur Herleitung der für den bestehenden Prozess erforderlichen Kompetenzen wurden in drei KMU leitfadengestützte Experteninterviews durchgeführt und inhaltsanalytisch ausgewertet. Darauf aufbauend wurde ein generisches Kompetenzmodell entwickelt, welches genutzt wurde, um die spezifischen Kompetenzfacetten der einzelnen prozessbeteiligten Tätigkeitsbereiche zu formulieren und in einem tätigkeitsbezogenen Kompetenzmodell $\mathrm{zu}$ verdichten. Zur Erstellung der prozessbezogenen Kompetenzprofile wurden die im tätigkeitsbezogenen Kompetenzmodell aufgeführten Kompetenzfacetten den einzelnen Prozessschritten zugeordnet. Abschließend erfolgte eine kommunikative Va- 
lidierung der entwickelten Kompetenzprofile. Dazu werden den Interviewten ihre Aussagen erneut vorgelegt, um diese zu akzeptieren, ggf. zu modifizieren oder zurückzuweisen. Besteht hier Zustimmung, wird dies als kommunikative Validierung der Interviewaussagen durch die Interviewten bezeichnet (Flick 2020). Im Rahmen des präsentierten Vorgehens bezieht sich die kommunikative Validierung auf das Überprüfen und Akzeptieren der entwickelten prozessbezogenen Kompetenzprofile durch die Interviewten.

\subsubsection{Instrumente}

Das in den gegenwartsbezogenen Interviews eingesetzte Instrumentarium kombiniert, angelehnt an die TAToo (Koch und Westhoff 2012), verschiedene Methoden der Anforderungsanalyse und umfasst einen strukturierten Interviewleitfaden sowie zwei integrierte Begleitinstrumente (Einschätzungsbogen zu den Arbeitsgestaltungsmerkmalen und Kompetenzinventar). Um den Prozessbezug während der Interviews herzustellen, wird außerdem eine mit der graphischen Spezifikationssprache BPMN (Business Process Model and Notation) erstellte Graphik des jeweiligen Arbeits- bzw. Geschäftsprozesses eingesetzt.

Das erste Modul entspricht der erfahrungsgeleitet-intuitiven Methode der Anforderungsanalyse (vgl. Abschn. 2.2). Mit Hilfe konkreter Interviewfragen werden zunächst die mit den einzelnen Prozessschritten verbundenen Tätigkeiten und Herausforderungen erfasst. Das zweite Modul beruht auf der arbeitsplatzanalytisch-empirischen Methode der Anforderungsanalyse. Hierbei werden die mit den Tätigkeiten verbundenen Aufgaben-, Wissens- und sozialen Merkmale unter Anwendung eines auf einer angepassten Version des WDQ (Stegmann et al. 2010) basierenden Einschätzungsbogens (Begleitinstrument 1) identifiziert. Der WDQ dient hier als Grundlage für diesen Analyseschritt, da es sich um ein häufig eingesetztes arbeitsanalytisches Verfahren handelt (Schaper 2019). Um die Dauer des Interviews angemessen zu gestalten, erfolgten die Erfassung und Analyse der Arbeitsgestaltungsmerkmale auf einer globalen Ebene. Daher werden nicht alle Inhalte des WDQ abgefragt, sondern eine Auswahl der für den betrieblichen Kontext relevantesten Merkmalsdimensionen betrachtet. Die jeweiligen Arbeitsgestaltungsmerkmale werden im Gegensatz zu Stegmann et al. (2010) nicht als positive Aussagen formuliert und über die Zustimmung bzw. Ablehnung der Interviewten zu diesen Aussagen auf einer fünfstufigen Likert-Skala $(1=$ stimme überhaupt nicht zu bis $5=$ stimme voll zu) erfasst, sondern in einer eigenen prozessbezogenen Fragestellung ermittelt. Hierbei sollen die Interviewten einschätzen, wie stark die Arbeitsgestaltungsmerkmale für den jeweils betrachteten Geschäfts- bzw. Arbeitsprozess ausgeprägt sind. Zu diesem Zweck wurde auch die in Stegmann et al. (2010) verwendete Antwortskala abgeändert und mit den Antwortkategorien $1=$ sehr gering bis $5=$ sehr hoch versehen. Da das präsentierte Vorgehen auf einem qualitativen Forschungsdesign basiert, stehen die individuellen Einschätzungen der Interviewten im Fokus. Daher wird die Antwortskala nicht dazu eingesetzt, um die Ausprägung der Arbeitsgestaltungsmerkmale quantitativ auszuwerten (z.B. über Mittelwertbildung), sondern um die Interviewten dabei zu unterstützen, ihre Einschätzungen zu den jeweils betrachteten Arbeitsgestaltungsmerkmalen zu konkretisieren und anhand von Praxisbeispielen zu veranschaulichen.

Wie in Abb. 1 dargestellt, werden im Einschätzungsbogen unter jedem Arbeitsgestaltungsmerkmal die prozessbezogene Fragestellung sowie die zur Einschätzung zu verwendende Likert-Skala aufgeführt. Das dritte Modul basiert wiederum auf der erfahrungsgeleitet-intuitiven Methode der Anforderungsanalyse. Hierbei werden mit Hilfe der CIT (Flanagan 1954) und entsprechenden, an die TAToo angelehnten, Interviewfragen eine im Prozess auftretende erfolgskritische Arbeitssituation sowie die dabei eingesetzten effektiven Verhaltensweisen erfasst. Die CIT wird hier angewendet, da es sich um eine weit verbreitete und bewährte Methode zur konkreten und verhaltensnahen Erfassung von Arbeitstätigkeiten sowie der damit verbundenen Anforderungen handelt (Koch 2010). Das vierte Modul gründet erneut auf der arbeitsplatzanalytisch-empirischen Methode der Anforderungsanalyse, wobei die für den betrachteten Prozess erforderlichen Kompetenzen aus einem Kompetenzinventar (Begleitinstrument 2) ausgewählt werden. Das Kompetenzinventar untergliedert sich in die vier Bereiche der beruflichen Handlungskompetenz und umfasst

\begin{tabular}{|c|c|c|c|c|c|c|}
\hline \multicolumn{7}{|c|}{ Arbeitsgestaltungsmerkmale } \\
\hline \multicolumn{7}{|c|}{ Autonomie } \\
\hline \multicolumn{7}{|c|}{$\begin{array}{l}\text { Wie hoch schätzen Sie bezogen auf Ihre Tätigkeiten im aktuellen } \\
\text { Prozess das Ausmaß ein, in dem Sie diese unabhängig koordinieren } \\
\text { können? }\end{array}$} \\
\hline $\begin{array}{l}\text { sehr } \\
\text { gering }\end{array}$ & 1 & 2 & 3 & 4 & 5 & $\begin{array}{l}\text { sehr } \\
\text { hoch }\end{array}$ \\
\hline \multicolumn{7}{|c|}{ Problemlösen } \\
\hline \multicolumn{7}{|c|}{$\begin{array}{l}\text { Wie hoch schätzen Sie bezogen auf Ihre Tätigkeiten im aktuellen } \\
\text { Prozess das Ausmaß ein, in dem Sie für Probleme kreative Lösungen } \\
\text { entwickeln müssen? }\end{array}$} \\
\hline $\begin{array}{l}\text { sehr } \\
\text { gering }\end{array}$ & 1 & 2 & 3 & 4 & 5 & $\begin{array}{l}\text { sehr } \\
\text { hoch }\end{array}$ \\
\hline \multicolumn{7}{|c|}{ Unterstützung innerhalb der Organisation } \\
\hline \multicolumn{7}{|c|}{$\begin{array}{l}\text { Wie hoch schätzen Sie bezogen auf Ihre Tätigkeiten im aktuellen } \\
\text { Prozess die Unterstützung von Vorgesetzten/KollegInnen ein? }\end{array}$} \\
\hline $\begin{array}{l}\text { sehr } \\
\text { gering }\end{array}$ & 1 & 2 & 3 & 4 & 5 & $\begin{array}{l}\text { sehr } \\
\text { hoch }\end{array}$ \\
\hline
\end{tabular}

Abb. 1 Ausschnitt aus dem Einschätzungsbogen zu den Arbeitsgestaltungsmerkmalen. (In Anlehnung an den WDQ, Stegmann et al. 2010) Fig. 1 Excerpt from the record sheet of the work design characteristics. (Based on the WDQ, Stegmann et al. 2010) 
Tab. 1 Ausschnitt aus dem Kompetenzinventar. (In Anlehnung an Carretero et al. 2017; Heyse 2010; Kauffeld 2010)

Table 1 Excerpt from the competence inventory. (Based on Carretero et al. 2017; Heyse 2010; Kauffeld 2010)

\begin{tabular}{|c|c|c|c|}
\hline \multicolumn{2}{|c|}{ Kompetenzen } & Beschreibung & Beispiel \\
\hline \multicolumn{4}{|c|}{ Fachkompetenz } \\
\hline 1. & Fachwissen & $\begin{array}{l}\text { Nutzung fachlicher und methodischer Kenntnisse (vgl. } \\
\text { Heyse 2010) }\end{array}$ & Produktkenntnisse, Prozesskenntnisse \\
\hline 2. & $\begin{array}{l}\text { Fachübergreifendes } \\
\text { Wissen }\end{array}$ & $\begin{array}{l}\text { Nutzung überfachlicher Allgemeinbildung (vgl. Heyse } \\
\text { 2010) }\end{array}$ & Kenntnis verschiedener Arbeitsmethoden \\
\hline \multicolumn{4}{|c|}{ Methodenkompetenz } \\
\hline 1. & Problemlösefähigkeit & $\begin{array}{l}\text { Anstoßen und Begleiten von Problemlöseprozessen (vgl. } \\
\text { Heyse 2010) }\end{array}$ & $\begin{array}{l}\text { Erkennen und Beseitigen von Produkt- } \\
\text { oder Maschinenmängeln }\end{array}$ \\
\hline 2. & Ganzheitliches Denken & $\begin{array}{l}\text { Wechselwirkungen des eigenen Handelns berücksichti- } \\
\text { gen (vgl. Heyse 2010) }\end{array}$ & Antizipieren wichtiger Arbeitshandlungen \\
\hline
\end{tabular}

Tab. 2 Stichprobe der Experteninterviews

Table 2 Sample of the expert interviews

\begin{tabular}{|c|c|c|c|}
\hline & KMU 1 & KMU 2 & KMU 3 \\
\hline Branche & Glasgroßhandel & Metallbau & Lebensmittelindustrie \\
\hline $\begin{array}{l}\text { Anzahl der } \\
\text { Beschäftigten }\end{array}$ & 41 & 65 & 200 \\
\hline $\begin{array}{l}\text { Interviewte } \\
\text { ExpertInnen }\end{array}$ & $\begin{array}{l}\text { Führungskraft Einkauf, } \\
\text { MitarbeiterIn Verkauf, Mitarbeiter- } \\
\text { In Lager }\end{array}$ & $\begin{array}{l}\text { Führungskraft Einkauf, MitarbeiterIn } \\
\text { Schweißerei, MitarbeiterIn Schweißauf- } \\
\text { sicht }\end{array}$ & $\begin{array}{l}\text { Führungskraft Qualitätsmanagement, } \\
\text { MitarbeiterInnen Qualitätskontrolle }\end{array}$ \\
\hline
\end{tabular}

darüber hinaus die digitale Kompetenz als fünften Kompetenzbereich.

Die im Kompetenzinventar aufgeführten Fach-, Methoden-, Sozial- und Selbstkompetenzen wurden auf Basis des Kompetenzatlas (Heyse und Erpenbeck 2009) und des KRI (Kauffeld 2010) abgeleitet, da beide Instrumente die Bereiche der beruflichen Handlungskompetenz umfassend und strukturiert abbilden. Die im Kompetenzinventar thematisierten Facetten der digitalen Kompetenz wurden auf Grundlage des DigComp 2.1 (Carretero et al. 2017) entwickelt, da es sich hier um ein in diesem Bereich vielfach eingesetzten Kompetenzinventars handelt, welches die Kernbereiche der digitalen Kompetenz sehr konkret und praxisnah aufführt (Vuorikari et al. 2016). Tab. 1 zeigt einen Ausschnitt aus dem Kompetenzinventar: Unter den einzelnen Kompetenzbereichen werden die jeweils zugehörigen Kompetenzen aufgelistet und definiert. Zur Veranschaulichung der Kompetenzen werden zusätzlich Praxisbeispiele aufgeführt. Im fünften Modul werden abschließend der Digitalisierungsstand im bestehenden Prozess sowie die Offenheit der Mitarbeitenden im Hinblick auf weitere digitale Veränderungen anhand von konkreten Interviewfragen erfasst. Hierbei soll beschrieben werden, welche digitalen Technologien bereits im Prozess implementiert wurden und wie die Mitarbeitenden darauf reagiert haben.

\subsubsection{Stichprobe}

Die Interviews wurden in drei KMU unterschiedlicher Branchen durchgeführt. Insgesamt fanden neun Interviews statt, wobei pro KMU jeweils zwei am Prozess beteiligte Mitarbeitende und eine Führungskraft befragt wurden. Tab. 2 gibt einen Überblick über die Branchen der drei KMU, die Beschäftigtenzahlen und die Positionen der befragten ExpertInnen. Die Interviews bezogen sich jeweils auf einen ausgewählten Arbeits- und Geschäftsprozess, der in der Zukunft digitalisiert werden soll. KMU 1 hat sich zum Ziel gesetzt, in seinen Bestandsmanagementprozess ein digitales System zu implementieren, welches Warenein- und -abgänge in Echtzeit erfasst. KMU 2 und 3 planen zukünftig Tablets an verschiedenen Stellen einzusetzen, um interne Kommunikationsstrukturen effektiver $\mathrm{zu}$ gestalten. Um die Schritte der gegenwartsbezogenen Analysephase möglichst konkret zu beschreiben, werden im Folgenden beispielhaft Ergebnisse aus dem Experteninterview mit einem am Bestandsmanagementprozess von KMU 1 beteiligten Einkäufer berichtet.

\subsubsection{Durchführung der gegenwartsbezogenen Experteninterviews}

Die Interviews dauerten ca. $60 \mathrm{~min}$, wobei von jedem Interview für die spätere inhaltsanalytische Auswertung eine Audioaufnahme angefertigt wurde. Während der Interviews wurde durchgängig auf die zuvor erstellte BPMNProzessgraphik Bezug genommen. In Modul 1 wurden die mit den gegenwärtigen Prozessschritten verbundenen Arbeitstätigkeiten sowie mögliche Herausforderungen bei deren Ausführung anhand der Prozessgraphik erfragt bzw. von den ExpertInnen beschrieben. Beispielsweise wurde in Be- 
zug auf den Bestandsmanagementprozess verdeutlicht bzw. als Herausforderung benannt, dass sich der Warenbestand manchmal schneller als erwartet reduziert und daher Planungsfähigkeiten besonders wichtig sind.

$\mathrm{Zu}$ Beginn von Modul 2 wurden zunächst die Arbeitsgestaltungsmerkmale des Einschätzungsbogens (Begleitinstrument 1) vorgestellt sowie die zu deren Einschätzung anzuwendende Likert-Skala genauer beschrieben. Anschließend schätzten die ExpertInnen die Ausprägungen der Arbeitsgestaltungsmerkmale in Bezug auf den jeweils betrachteten Prozess ein und veranschaulichten dies anhand von Praxisbeispielen. Beispielsweise wurde im Bestandsmanagementprozess das Merkmal Autonomie sehr hoch eingeschätzt, da durch den Einkauf eigenständig entschieden werden kann, wann Bestandsprüfungen vorgenommen werden sollen.

In Modul 3 wurde zunächst der Begriff der erfolgskritischen Arbeitssituation geklärt: Es sollte eine Situation erinnert werden, in der die Mitarbeitenden besonders geschickt sein mussten, um zu einem erfolgreichen Arbeitsergebnis zu gelangen. Beispielsweise wurde im Hinblick auf den Bestandsmanagementprozess die Situation beschrieben, dass Kunden oftmals kurzfristige Bestelländerungen vornehmen und deshalb zusätzliche Glasware schnellstmöglich beschafft werden muss. Als eine besonders effektive und wichtige Verhaltensweise wurde hier der Informationsaustausch mit dem Verkauf und dem Versand beschrieben.

$\mathrm{Zu}$ Beginn von Modul 4 wurden die im Kompetenzinventar (Begleitinstrument 2) aufgeführten Kompetenzen vorgestellt. Die ExpertInnen sollten anschließend die für den jeweils betrachteten Prozess besonders relevanten Kompetenzen auswählen und dies anhand von Praxisbeispielen verdeutlichen. Beispielsweise wurde im Hinblick auf den Bestandsmanagementprozess betont, dass Fachwissen (z.B. Materialkenntnisse) erforderlich ist, um Warenbestellungen durchzuführen.

In Modul 5 sollte abschließend durch die ExpertInnen beschrieben werden, welche digitalen Veränderungen bereits im betrachteten Prozess stattgefunden haben und wie die Mitarbeitenden darauf reagiert haben. Beispielsweise wurde in Bezug auf den Bestandsmanagementprozess in KMU 1 erwähnt, dass bereits ein digitales Dokumentensystem implementiert wurde, um Einkaufs- und Verkaufsdaten zu dokumentieren und zu archivieren. Die Reaktionen der Mitarbeitenden auf die Implementierung dieses Systems wurden als sehr positiv wahrgenommen, da bspw. Einkaufsrechnungen nun strukturierter und übersichtlicher abgelegt werden können.

\subsubsection{Auswertung der gegenwartsbezogenen Experteninterviews}

Die Interviews wurden wörtlich transkribiert und unter Anwendung der Software MAXQDA 12 anhand eines theoriegeleiteten Kategoriensystems inhaltsanalytisch ausgewertet (Mayring 2010). Im Sinne eines deduktiven Vorgehens wurden die Interviewtextstellen hierbei den KompetenzKategorien eines zuvor entwickelten Kategoriensystems zugeordnet. Das Kategoriensystem basiert auf dem im vorherigen Abschnitt beschriebenen Kompetenzinventar (vgl. Tab. 1) und umfasst daher Kompetenzen aus dem KRI (Kauffeld 2010), dem Kompetenzatlas (Heyse und Erpenbeck 2009) und dem DigComp 2.1 (Carretero et al. 2017). Diese Kompetenzen bilden die Subebene des Kategoriensystems und sind den fünf übergeordneten Bereichen der Fach-, Methoden-, Sozial-, Selbst- und der digitalen Kompetenz zugeordnet. Falls bestimmte Interviewtextstellen nicht eindeutig in das Kategoriensystem eingeordnet werden konnten, wurden induktiv neue Kategorien gebildet. Im Sinne eines iterativen Vorgehens wurden die Kategorien so lange überarbeitet, bis alle relevanten Interviewtextstellen zweifelsfrei zugeordnet werden konnten (Mayring 2010). Um zu überprüfen, ob die Interviewtextstellen tatsächlich eindeutig in das Kategoriensystem eingeordnet werden konnten, wurde die Interrater-Reliabilität bestimmt. Hierzu erfolgte die Berechnung des Cohens-Kappa-Koeffizienten (Cohen 1960), welcher das Ausmaß angibt, in dem zwei voneinander unabhängige BeurteilerInnen gleiche Textstellen denselben Kategorien zuordnen (Krüger und Riemeier 2014). Zur Berechnung des Koeffizienten wurde eine Stichprobe herangezogen, die $25 \%$ aller zugordneten Textstellen $(N=292)$ aus den gegenwartsbezogenen Experteninterviews umfasst. Für die Hauptebene des Kategoriensystems ergab sich ein Kappa-Wert von 0,766 und für die Subebene ein Wert von 0,725.

\subsubsection{Erstellung gegenwartsbezogener Kompetenzmodelle}

Mit Bezug auf das Kategoriensystem wurde zunächst ein generisches Kompetenzmodell entwickelt, dessen Kompetenzen für alle drei KMU relevant sind. Dazu wurden auf Grundlage der mit den Interviewtextstellen angereicherten Kategorien des Kategoriensystems unternehmensübergreifende Kompetenzen formuliert und definiert. Basierend auf dem generischen Kompetenzmodell wurden für die prozessrelevanten Tätigkeitsbereiche der drei KMU schließlich tätigkeitsbezogene Kompetenzmodelle entwickelt, indem die Kompetenzen des generischen Kompetenzmodells um tätigkeitsspezifische Kompetenzfacetten erweitert wurden. Die Kompetenzfacetten wurden auf Grundlage der jeweils zugeordneten Interviewtextstellen formuliert. 


\subsubsection{Erstellung gegenwarts- und prozessbezogener Kompetenzprofile}

Anhand der tätigkeitsbezogenen Kompetenzmodelle wurden schließlich die gegenwarts- und prozessbezogenen Kompetenzprofile hergeleitet, indem die in den tätigkeitsbezogenen Kompetenzmodellen aufgeführten Kompetenzen den einzelnen Schritten des jeweils betrachteten Arbeitsbzw. Geschäftsprozesses zugeordnet wurden. Die Zuordnung wurde durch den/die InterviewerIn auf Basis der Interviewaussagen vorgenommen.

Daraufhin erfolgte die kommunikative Validierung der gegenwarts- und prozessbezogenen Kompetenzprofile, wobei die in den Interviews befragten ExpertInnen unter Hinzunahme des tätigkeitsbezogenen Kompetenzmodells überprüften, ob alle relevanten Kompetenzen in ihrem prozessbezogenen Kompetenzprofil abgebildet waren. Anschließend bewerteten sie bei den Kompetenzen mithilfe eines Einstufungsschlüssels den erforderlichen SollAusprägungsgrad (vgl. Tab. 3). Der Einstufungsschlüssel wurde in Anlehnung an den im Rahmen des CMT entwickelten und eingesetzten Einstufungsschlüssels (Decius und Schaper 2017; vgl. Abschn. 2.3) konzipiert und umfasst insgesamt fünf Stufen. Jede Stufe repräsentiert einen bestimmten Kompetenz-Ausprägungsgrad und untergliedert sich in zwei Bewertungsaspekte. Der erste Aspekt bezieht sich auf den Kenntnis- und Beherrschungsgrad der Mitarbeitenden und wird somit zur Einstufung ihrer Fach- und Methodenkompetenzen herangezogen. Der zweite Aspekt dient schließlich zur Einschätzung der Sozial- und Selbstkompetenzen. Da die digitale Kompetenz hier als Querschnittskompetenz aufgefasst wird, wird je

Tab. 3 Einstufungsschlüssel zur Festlegung der Soll-Kompetenzen. (In Anlehnung an Decius und Schaper 2017)

Table 3 Rating key for determining the required competences. (Based on Decius and Schaper 2017)

\begin{tabular}{|c|c|}
\hline Stufe & Beschreibung \\
\hline \multirow[t]{2}{*}{0} & Keine Kenntnisse/kein Beherrschungsgrad \\
\hline & Kein angemessenes Sozialverhalten/Selbstmanagement \\
\hline \multirow[t]{2}{*}{1} & Grundkenntnisse/grundlegender Beherrschungsgrad \\
\hline & Grundlegendes Sozialverhalten/Selbstmanagement \\
\hline \multirow[t]{2}{*}{2} & $\begin{array}{l}\text { Anwenderkenntnisse/sicherer Beherrschungsgrad in Stan- } \\
\text { dardsituationen }\end{array}$ \\
\hline & $\begin{array}{l}\text { Angemessenes Sozialverhalten/Selbstmanagement in } \\
\text { Standardsituationen }\end{array}$ \\
\hline \multirow[t]{2}{*}{3} & $\begin{array}{l}\text { Vertiefte Kenntnisse/sicherer Beherrschungsgrad in ab- } \\
\text { weichenden Situationen }\end{array}$ \\
\hline & $\begin{array}{l}\text { Angemessenes Sozialverhalten/Selbstmanagement in } \\
\text { abweichenden Situationen }\end{array}$ \\
\hline \multirow[t]{2}{*}{4} & $\begin{array}{l}\text { Sehr detaillierte Kenntnisse/sicherer Beherrschungsgrad in } \\
\text { neuartigen Situationen }\end{array}$ \\
\hline & $\begin{array}{l}\text { Angemessenes Sozialverhalten/Selbstmanagement in } \\
\text { neuartigen Situationen }\end{array}$ \\
\hline
\end{tabular}

nach betrachteter Kompetenzdimension der entsprechende Bewertungsaspekt ausgewählt und eingestuft.

\subsection{Ergebnisse}

Das zentrale Ergebnis der gegenwartsbezogenen Analysephase sind die gegenwarts- und prozessbezogenen Kompetenzprofile, welche die für einen bestimmten Tätigkeitsbereich erforderlichen Kompetenzen in Bezug auf einzelne Prozessschritte aufzeigen. Wie in Abschn. 3.1 aufgeführt, wurde dazu zunächst ein generisches Kompetenzmodell entwickelt, welches genutzt wurde, um tätigkeitsspezifische Kompetenzfacetten zu formulieren und in einem entsprechenden tätigkeitsbezogenen Kompetenzmodell zusammenzufassen. Zur Entwicklung der gegenwarts- und prozessbezogenen Kompetenzprofile wurden die tätigkeitsspezifischen Kompetenzfacetten den einzelnen Prozessschritten zugeordnet.

\subsubsection{Gegenwartsbezogene Kompetenzmodelle}

Das generische Kompetenzmodell basiert auf dem in der inhaltsanalytischen Auswertung eingesetzten Kategoriensystem. Wie in Tab. 4 beispielhaft aufgeführt, untergliedert sich die erste Ebene in die fünf übergeordneten Bereiche der Fach-, Methoden-, Sozial- und Selbstkompetenz (berufliche Handlungskompetenz) sowie der digitalen Kompetenz. Auf der zweiten Ebene werden die zu den übergeordneten Kompetenzbereichen zugehörigen Kompetenzen aufgeführt und definiert. Beispielsweise geht aus der Beschreibung der Kompetenz Anwendung von Fachwissen hervor, dass sich diese auf die Nutzung des für die Tätigkeitsausführung relevanten methodischen und fachlichen Wissens bezieht.

Auf Grundlage des generischen Kompetenzmodells wurden anschließend die tätigkeitsbezogenen Kompetenzmodelle entwickelt. Tab. 5 zeigt einen Ausschnitt aus dem tätigkeitsbezogenen Kompetenzmodell des am Bestandsmanagementprozess von KMU 1 beteiligten Einkäufers. Daneben werden beispielhafte Textstellen aus dem Experteninterview mit dem Einkäufer aufgeführt, anhand derer die spezifischen Kompetenzfacetten hergeleitet und formuliert wurden. Es wird bspw. deutlich, dass der Einkäufer zur erfolgreichen Bewältigung seiner Tätigkeiten spezifisches Fachwissen anwenden muss, welches sich insbesondere auf Materialkenntnisse bezieht. Ebenfalls wichtig ist das ganzheitliche Denken, welches das Antizipieren zukünftiger Bestellanforderungen inkludiert. Auch die Kommunikationsfähigkeit, welche das Anpassen der eigenen Ausdrucksweise umfasst sowie die Verantwortungsübernahme, welche sich insbesondere auf das gewissenhafte und ordentliche Führen von Bestandslisten bezieht, sind für eine erfolgreiche Tätigkeitsausführung des Einkäufers erforderlich. Darüber hinaus ist es notwendig, dass der Einkäufer den Umgang 
Tab. 4 Ausschnitt aus dem generischen Kompetenzmodell der drei KMU

Table 4 Excerpt from the generic competence model of the three SMEs

\begin{tabular}{ll}
\hline Kompetenzen & Beschreibung \\
\hline $\begin{array}{l}\text { Fachkompetenz } \\
\text { Anwendung von Fachwissen }\end{array}$ & Nutzung von fachlichem und methodischem Wissen zur Ausführung der Arbeitsaufgaben (vgl. Heyse 2010) \\
$\begin{array}{l}\text { Methodenkompetenz } \\
\text { Ganzheitliches Denken }\end{array}$ & Denken in der gesamten Prozesskette, Folgebewusstsein (vgl. Heyse 2010; Kauffeld 2010) \\
$\begin{array}{l}\text { Sozialkompetenz } \\
\text { Kommunikationsfähigkeit }\end{array}$ & Zielgruppengerechte Ausdrucksweise (vgl. Heyse 2010) \\
$\begin{array}{l}\text { Selbstkompetenz } \\
\text { Verantwortungsübernahme }\end{array}$ & Gründliches und umsichtiges Ausführen der Arbeitsaufgaben (vgl. Heyse 2010; Kauffeld 2010) \\
$\begin{array}{l}\text { Digitale Kompetenz } \\
\text { Umgang mit digitalen Daten }\end{array}$ & Systematisches Suchen, Filtern, Evaluieren und Verarbeiten digitaler Daten (vgl. Carretero et al. 2017) \\
\hline
\end{tabular}

Tab. 5 Ausschnitt aus einem tätigkeitsbezogenen Kompetenzmodell

Table 5 Excerpt from an activity-related competence model

\begin{tabular}{|c|c|c|}
\hline Kompetenzen & Facetten (beispielhaft) & Interviewtextstelle \\
\hline \multicolumn{3}{|l|}{ Fachkompetenz } \\
\hline Anwendung von Fachwissen & Materialkenntnisse & „Fachwissen über Materialkenntnisse, das brauche ich.“ \\
\hline \multicolumn{3}{|l|}{ Methodenkompetenz } \\
\hline Ganzheitliches Denken & $\begin{array}{l}\text { Antizipieren zukünftiger Bestellan- } \\
\text { forderungen }\end{array}$ & $\begin{array}{l}\text { „Also es geht einmal darum, dass natürlich der Bestand nicht auf } 0 \\
\text { läuft.“ }\end{array}$ \\
\hline \multicolumn{3}{|l|}{ Sozialkompetenz } \\
\hline Kommunikationsfähigkeit & $\begin{array}{l}\text { Anpassen der eigenen Ausdruckswei- } \\
\text { se }\end{array}$ & „Vielleicht nicht zu viel Fachjargon verwenden." \\
\hline \multicolumn{3}{|l|}{ Selbstkompetenz } \\
\hline Verantwortungsübernahme & $\begin{array}{l}\text { Gewissenhaftes und ordentliches } \\
\text { Führen von Bestandslisten }\end{array}$ & „Verlässliche Listen über Bestände führen.“ \\
\hline \multicolumn{3}{|l|}{ Digitale Kompetenz. } \\
\hline Umgang mit digitalen Daten & $\begin{array}{l}\text { Systematisches Evaluieren digitaler } \\
\text { Einkaufsdaten }\end{array}$ & $\begin{array}{l}\text { „Die Anforderungen wären, dass entsprechende Datenauswertungs- } \\
\text { modelle hinterlegt werden müssen." }\end{array}$ \\
\hline
\end{tabular}

mit digitalen Daten, wie bspw. das systematische Evaluieren digitaler Daten, beherrscht.

\subsubsection{Gegenwarts- und prozessbezogene Kompetenzprofile}

Zur Entwicklung der gegenwarts- und prozessbezogenen Kompetenzprofile wurden die im tätigkeitsbezogenen Kompetenzmodell aufgeführten Kompetenzfacetten den einzelnen Prozessschritten des jeweils betrachteten Tätigkeitsbereichs zugeordnet. Um die zugeordneten Kompetenzen wei- ter zu konkretisieren, werden dahinter jeweils die spezifischen Kompetenzfacetten sowie der erforderliche Soll-Ausprägungsgrad abgebildet. Tab. 6 zeigt einen Ausschnitt aus dem gegenwarts- und prozessbezogenen Kompetenzprofil des am Bestandsmanagementprozess von KMU 1 beteiligten Einkäufers. Hier wird deutlich, dass bei beiden dargestellten Prozessschritten ein sehr detailliertes Fachwissen erforderlich ist (Stufe 4 im Einstufungsschlüssel).

Bei Prozessschritt 1 ist zudem ein sehr detailliertes Erfahrungswissen notwendig. Ebenfalls hohe Anforderungen werden bei beiden Prozessschritten an das ganzheitliche

Tab. 6 Ausschnitt aus einem gegenwarts- und prozessbezogenen Kompetenzprofil

Table 6 Excerpt from a current- and process-related competence profile

\begin{tabular}{lll}
\hline $\begin{array}{l}\text { Prozessschritt 1: } \\
\text { Bedarf ermitteln }\end{array}$ & Soll & $\begin{array}{l}\text { Prozessschritt 2: } \\
\text { Einkäufe planen }\end{array}$ \\
\hline $\begin{array}{l}\text { Anwendung von Fachwissen: } \\
\text { Materialkenntnisse }\end{array}$ & 4 & $\begin{array}{l}\text { Anwendung von Fachwissen: } \\
\text { Materialkenntnisse } \\
\text { Anwendung von Erfahrungswissen: }\end{array}$ \\
$\begin{array}{l}\text { Kenntnis von Bestellhistorien } \\
\text { Ganzheitliches Denken: }\end{array}$ & 4 & $\begin{array}{l}\text { Antizipieren zukünftiger Bestellanforderungen } \\
\text { Kommunikationsfähigkeit: } \\
\text { Antizipieren zukünftiger Bestellanforderungen }\end{array}$ \\
\hline
\end{tabular}


Denken gestellt, da hier ein Kompetenzgrad von 3 vorausgesetzt wird. Abschließend ist zu erkennen, dass bei Prozessschritt 2, mit einem erforderlichen Kompetenzgrad von 4, sehr hohe Anforderungen an die Kommunikationsfähigkeit gestellt werden. Die gegenwarts- und prozessbezogenen Kompetenzprofile dienen in der nachfolgenden zukunftsbezogenen Analysephase als Orientierungsgrundlage, um zukünftige Entwicklungsbedarfe ableiten zu können.

\section{Zukunftsbezogene Analysephase}

Das Ziel der zukunftsbezogenen Analysephase ist die Entwicklung von zukunfts- und prozessbezogenen Kompetenzprofilen, welche die für den veränderten, nun digitalisierten Prozess erforderlichen Kompetenzen aufführen. Auf dieser Grundlage ist es möglich, zukünftige Entwicklungsbedarfe zu identifizieren.

\subsection{Methode}

Zur Bestimmung der für den digitalisierten Prozess erforderlichen Kompetenzen wurden in den drei KMU nochmals leitfadengestützte Experteninterviews durchgeführt und inhaltsanalytisch ausgewertet. Anschließend wurde erneut das generische Kompetenzmodell (vgl. Abschn. 3.2.1) herangezogen, um zunächst die spezifischen Kompetenzfacetten für die einzelnen am Prozess beteiligten Tätigkeitsbereiche $\mathrm{zu}$ formulieren und in entsprechenden zukunftsund tätigkeitsbezogenen Kompetenzmodellen zusammenzufassen. Zur anschließenden Erstellung der zukunfts- und prozessbezogenen Kompetenzprofile wurden die in den tätigkeitsbezogenen Kompetenzmodellen aufgeführten Kompetenzfacetten den einzelnen Schritten des digitalisierten Prozesses zugeordnet. Abschließend erfolgte auch hier eine kommunikative Validierung der resultierenden zukunftsund prozessbezogenen Kompetenzprofile.

\subsubsection{Instrumente}

In den zukunftsbezogenen Experteninterviews wurden grundsätzlich dieselben Befragungs- und Begleitinstrumente wie in den gegenwartsbezogenen Experteninterviews eingesetzt (vgl. Abschn. 3.1.1). Das Instrumentarium wurde jedoch an bestimmten Stellen an den zukunftsbezogenen Analysefokus angepasst. Daher wurden aus dem Interviewleitfaden alle Fragestellungen entfernt, die aus der prospektiven Perspektive heraus nur schwer beantwortet werden können (z. B. zukünftige Resultate kritischer Ereignisse) oder die keine inhaltliche Relevanz mehr mit Blick auf die zukunftsbezogene Analyse aufweisen (z.B. Interviewmodul 5). Um den Prozessbezug auch während der zukunftsbezogenen Experteninterviews herzustellen, wur- de das Instrumentarium nun mit Bezug auf eine Graphik des digitalisierten Prozesses angewendet. Der digitalisierte Prozess wurde vorab von den drei KMU in Eigenworkshops papierbasiert entwickelt und anschließend durch die wissenschaftlichen MitarbeiterInnen des Projekts in eine BPMN-Darstellung überführt.

\subsubsection{Stichprobe}

In den zukunftsbezogenen Interviews wurden dieselben ExpertInnen wie in der gegenwartsbezogenen Analysephase befragt (vgl. Abschn. 3.1.2). Um die Schritte der zukunftsbezogenen Analysephase möglichst konkret zu beschreiben, werden im Folgenden erneut beispielhaft Ergebnisse aus dem Experteninterview mit dem am zukünftigen Bestandsmanagementprozess von KMU 1 beteiligten Einkäufer aufgeführt.

\subsubsection{Durchführung der zukunftsbezogenen Experteninterviews}

Die zukunftsbezogenen Experteninterviews dauerten aufgrund der Anpassung des Instrumentariums ca. $45 \mathrm{~min}$. Auch hier wurde für die spätere inhaltsanalytische Auswertung von jedem Interview eine Audioaufnahme angefertigt.

In Modul 1 wurden nun die mit den neuen Prozessschritten verbundenen Tätigkeiten sowie mögliche Herausforderungen bei deren Ausführung mit Bezugnahme auf die Graphik des digitalisierten Prozesses erfragt. Beispielsweise wurde im Hinblick auf den zukünftigen Bestandsmanagementprozess verdeutlicht, dass unerwartete Reduzierungen des Bestands an Glasware durch das digitale System viel besser antizipiert werden können und diese Herausforderung nun viel einfacher zu bewältigen ist.

In Modul 2 wurden die Arbeitsgestaltungsmerkmale des digitalisierten Prozesses wieder unter Anwendung des Einschätzungsbogens (vgl. Abschn. 3.1.1) ermittelt. Die ExpertInnen erläuterten auch hier ihre Einschätzungen anhand von Praxisbeispielen. Beispielsweise wurde das Merkmal Autonomie im zukünftigen Bestandsmanagementprozess hoch eingeschätzt, da die Arbeitsplanung weiterhin eigenständig durch den Einkäufer erledigt werden muss. Das digitale System stellt dafür alle relevanten Informationen zur Verfügung.

In Modul 3 wurde die im gegenwartsbezogenen Interview beschriebene erfolgskritische Arbeitssituation erneut aufgegriffen: Es sollte überlegt werden, welche Verhaltensweisen nun zur Bewältigung derselben Situation im digitalisierten Prozess besonders effektiv sind. Beispielsweise wurde in Bezug auf den zukünftigen Bestandsmanagementprozess von KMU 1 betont, dass für die Umsetzung kurzfristiger Bestelländerungen nicht mehr primär der Informationsaustausch mit dem Versand erfolgsentscheidend sein 
wird, sondern vielmehr die Fähigkeit, benötigte Produktinformationen im digitalen System zu recherchieren.

In Modul 4 wurden die für den digitalisierten Prozess erforderlichen Kompetenzen erneut unter Anwendung des Kompetenzinventars (vgl. Abschn. 3.1.1) identifiziert. Die ExpertInnen sollten ihre Einschätzungen wieder anhand von Praxisbeispielen verdeutlichen. So wurde im Hinblick auf den zukünftigen Bestandsmanagementprozess betont, dass u. a. die Fähigkeit wichtig wird, die zur Bedarfsermittlung relevanten Informationen im digitalen System herauszufiltern und zu evaluieren.

\subsubsection{Auswertung der zukunftsbezogenen Experteninterviews}

Die zukunftsbezogenen Interviews wurden ebenfalls wörtlich transkribiert und gleichermaßen unter Anwendung des bereits in der gegenwartsbezogenen Analysephase eingesetzten Kategoriensystems (vgl. Abschn. 3.1.4) inhaltsanalytisch ausgewertet. Um sicherzugehen, dass auch die Textstellen der zukunftsbezogenen Experteninterviews eindeutig in das Kategoriensystem eingeordnet werden konnten, wurde erneut die Interrater-Reliabilität ermittelt. Zur Berechnung des Cohens-Kappa-Koeffizienten wurde eine Stichprobe herangezogen, die $25 \%$ aller zugeordneten Textstellen der zukunftsbezogenen Experteninterviews $(N=187)$ umfasste. Für die Hauptebene des Kategoriensystems ergab sich ein Kappa-Wert von 0,767 und für die Subebene ein Wert von 0,721 .

\subsubsection{Erstellung zukunftsbezogener Kompetenzmodelle}

Zur Erstellung des zukunftsbezogenen, generischen Kompetenzmodells wurde erneut das bereits in der gegenwartsbezogenen Analysephase eingesetzte Kategoriensystem herangezogen, um auf Grundlage der nun mit den Textstellen aus den zukunftsbezogenen Interviews angereicherten Kompetenz-Kategorien weitere unternehmensübergreifen- de Kompetenzen zu definieren. Die zukunfts- und tätigkeitsbezogenen Kompetenzmodelle wurden ebenfalls nach dem bereits in der gegenwartsbezogenen Analysephase beschriebenen Vorgehen entwickelt (vgl. Abschn. 3.1.5).

\subsubsection{Erstellung zukunfts- und prozessbezogener Kompetenzprofile}

Die Erstellung der zukunfts- und prozessbezogenen Kompetenzprofile erfolgte analog zu dem in der gegenwartsbezogenen Analysephase angewendeten Vorgehen. Dabei wurden die in den zukunfts- und tätigkeitsbezogenen Kompetenzmodellen aufgeführten Kompetenzfacetten den einzelnen Schritten des nun digitalisierten Prozesses zugeordnet. Anschließend erfolgte gleichermaßen eine kommunikative Validierung der Kompetenzprofile (vgl. Abschn. 3.1.6).

\subsection{Ergebnisse}

Das Hauptergebnis der zukunftsbezogenen Analysephase stellen die zukunfts- und prozessbezogenen Kompetenzprofile dar. Diese führen die für einen bestimmten Tätigkeitsbereich erforderlichen Kompetenzen in Bezug auf die einzelnen Schritte des zukünftigen, digitalisierten Arbeitsbzw. Geschäftsprozesses auf.

\subsubsection{Zukunftsbezogene Kompetenzmodelle}

Da in den Arbeits- bzw. Geschäftsprozessen der drei KMU vorwiegend niederschwellige Digitalisierungsmaßnahmen vorgenommen wurden und sich die Aufgabenprofile der Mitarbeitenden daher nicht stark verändert haben, blieben die Kompetenzen des generischen Kompetenzmodells auch für den digitalisierten Prozess bestehen (vgl. Tab. 4). Somit wurde das generische Kompetenzmodell aus der gegenwartsbezogenen Analysephase (vgl. Abschn. 3.2.1) erneut verwendet, um für die an den digitalisierten Prozessen beteiligten Tätigkeitsbereiche entsprechende zukunfts- und

Tab. 7 Ausschnitt aus einem zukunfts- und tätigkeitsbezogenen Kompetenzmodell

Table 7 Excerpt from a future- and activity-related competence model

\begin{tabular}{|c|c|c|}
\hline Kompetenzen & Facetten (beispielhaft) & Interviewtextstelle \\
\hline \multicolumn{3}{|l|}{ Digitale Kompetenz } \\
\hline $\begin{array}{l}\text { Erzeugen digitaler } \\
\text { Daten }\end{array}$ & $\begin{array}{l}\text { Bestellinformationen in das digitale } \\
\text { System eintragen }\end{array}$ & $\begin{array}{l}\text { "[...] dass diese Datenerfassung jetzt nicht mehr analog von Liste zu Liste } \\
\text { händisch übertragen wird, sondern direkt in die Datenbank eingegeben } \\
\text { wird." }\end{array}$ \\
\hline $\begin{array}{l}\text { Umgang mit digitalen } \\
\text { Daten }\end{array}$ & $\begin{array}{l}\text { Systematisches Evaluieren digitaler } \\
\text { Einkaufsdaten }\end{array}$ & $\begin{array}{l}\text { „Die Anforderungen wären, dass entsprechende Datenauswertungsmodelle } \\
\text { hinterlegt werden müssen." }\end{array}$ \\
\hline $\begin{array}{l}\text { Digitale Kommunika- } \\
\text { tion und Zusammenar- } \\
\text { beit }\end{array}$ & $\begin{array}{l}\text { Informationsaustausch mit internen } \\
\text { Bereichen unter Einsatz des digitalen } \\
\text { Systems }\end{array}$ & $\begin{array}{l}\text { „Es geht darum, Informationsmengen zu selektieren, aufzubereiten und zur } \\
\text { Verfügung zu stellen.“ }\end{array}$ \\
\hline $\begin{array}{l}\text { Problemlösen mittels } \\
\text { digitaler Technologien }\end{array}$ & $\begin{array}{l}\text { Kreativer Einsatz des digitalen Sys- } \\
\text { tems, um Lösungen bei Lieferengpäs- } \\
\text { sen zu finden }\end{array}$ & $\begin{array}{l}\text { „Für die Entwicklung von Lösungen benötige ich schnell Informationen zu } \\
\text { vergangenen Bestellungen und Einkaufspreisen, welche nun im digitalen } \\
\text { System abgerufen werden können." }\end{array}$ \\
\hline
\end{tabular}


tätigkeitsbezogene Kompetenzmodelle zu entwickeln. Hierbei wurden insbesondere die tätigkeitsbezogenen $\mathrm{Fa}-$ cetten der digitalen Kompetenz inhaltlich angereichert.

In Tab. 7 wird bspw. deutlich, dass der Einkäufer aus KMU 1 zukünftig in der Lage sein muss, Bestellinformationen (z.B. Einkaufspreise, Lieferanteninformationen) in das digitale System einzutragen und systematisch zu evaluieren. Darüber hinaus wird von ihm zukünftig gefordert, diese digitalen Informationen für einen zielführenden Austausch mit anderen internen Bereichen aufzubereiten (z. B. Informationsselektion) und diese zur Lösung von Problemen, wie bspw. Lieferengpässen, heranzuziehen.

\subsubsection{Zukunfts- und prozessbezogene Kompetenzprofile}

Zur Entwicklung der zukunfts- und prozessbezogenen Kompetenzprofile wurden die in den zukunfts- und tätigkeitsbezogenen Kompetenzmodellen aufgeführten Kompetenzfacetten den zukünftigen Prozessschritten des jeweiligen Tätigkeitsbereichs zugeordnet. Der Aufbau und die Anwendungsmöglichkeiten der zukunfts- und prozessbezogenen Kompetenzprofile werden im Folgenden beispielhaft anhand des Kompetenzprofils des am digitalisierten Bestandsmanagementprozess von KMU 1 beteiligten Einkäufers erläutert (vgl. Tab. 8).

Die zukunfts- und prozessbezogenen Kompetenzprofile führen für jeden Tätigkeitsbereich auf, welche Kompetenzen zur Ausführung der einzelnen Prozessschritte im digitalisierten Prozess wichtig sind. Die abgebildeten Kompetenzen werden ebenso durch die jeweils zugehörigen Kompetenzfacetten und Soll-Ausprägungsgrade spezifiziert. Darüber hinaus werden neben den zukunftsbezogenen Soll-Ausprägungsgraden die in der gegenwartsbezogenen Analysephase identifizierten Soll-Ausprägungsgrade als Vergleichs- basis aufgeführt. Zusätzlich sind daneben die Ist-Kompetenzgrade der Mitarbeitenden dargestellt (vgl. Tab. 8).

Anhand der zukunfts- und prozessbezogenen Kompetenzprofile lassen sich Kompetenzentwicklungsbedarfe auf Ebene einzelner Mitarbeitender und Tätigkeitsbereiche sowie auf Ebene bestimmter Mitarbeitendengruppen ableiten. Zur Erfassung der Entwicklungsbedarfe eines/ einer einzelnen Mitarbeitenden erfolgt pro Prozessschritt ein Abgleich seiner/ihrer Ist-Kompetenzgrade mit den zukünftig erforderlichen Soll-Kompetenzgraden. Liegen hier Differenzen vor, lassen sich daraus entsprechende Entwicklungsbedarfe ableiten. Soll der Entwicklungsbedarf eines bestimmten Tätigkeitsbereichs ermittelt werden, erfolgt pro Prozessschritt ein Abgleich der aktuell und der zukünftig geforderten Soll-Kompetenzgrade: Werden für dieselbe Kompetenz im zukünftigen Prozess höhere Kompetenzgrade gefordert, entstehen dort möglicherweise Entwicklungsbedarfe. Der Entwicklungsbedarf bestimmter Mitarbeitendengruppen lässt sich anhand einer auf Häufigkeitseinstufungen beruhenden Auswertung der prozessbezogenen Kompetenzprofile identifizieren: Werden bestimmte Kompetenzen im zukünftigen, digitalisierten Prozess (aufgeführt in den zukunfts- und prozessbezogenen Kompetenzprofilen) viel häufiger als im aktuellen Prozess gefordert (aufgeführt in den gegenwarts- und prozessbezogenen Kompetenzprofilen), gewinnen diese an Bedeutung und sollten daher weiterentwickelt werden.

\subsubsection{Kompetenzentwicklungsbedarf auf Ebene einzelner Mitarbeitender und Tätigkeitsbereiche}

Um die Entwicklungsbedarfe einzelner Mitarbeitender prospektiv und prozessbezogen abzuleiten, wurden pro Prozessschritt die im zukunfts- und prozessbezogenen

Tab. 8 Ausschnitt aus einem zukunfts- und prozessbezogenen Kompetenzprofil

Table 8 Excerpt from a future- and process-related competence profile

\begin{tabular}{|c|c|c|c|c|c|c|c|}
\hline $\begin{array}{l}\text { Prozessschritt 1: } \\
\text { Bedarf ermitteln }\end{array}$ & Soll I & Soll II & Ist & $\begin{array}{l}\text { Prozessschritt 2: } \\
\text { Einkäufe planen }\end{array}$ & Soll I & Soll II & Ist \\
\hline $\begin{array}{l}\text { Anwendung von Fachwissen: } \\
\text { Materialkenntnisse }\end{array}$ & 4 & 2 & 4 & $\begin{array}{l}\text { Anwendung von Fachwissen: } \\
\text { Materialkenntnisse }\end{array}$ & 4 & 3 & 4 \\
\hline $\begin{array}{l}\text { Anwendung von Erfahrungswissen: } \\
\text { Kenntnis von Bestellhistorien }\end{array}$ & 4 & 2 & 4 & $\begin{array}{l}\text { Ganzheitliches Denken: } \\
\text { Antizipieren zukünftiger Bestel- } \\
\text { lanforderungen }\end{array}$ & 3 & 3 & 3 \\
\hline $\begin{array}{l}\text { Ganzheitliches Denken: } \\
\text { Antizipieren zukünftiger Bestellan- } \\
\text { forderungen }\end{array}$ & 3 & 3 & 3 & $\begin{array}{l}\text { Kommunikationsfähigkeit: } \\
\text { Anpassen der eigenen } \\
\text { Ausdrucksweise }\end{array}$ & 4 & 2 & 4 \\
\hline $\begin{array}{l}\text { Erzeugen digitaler Daten: } \\
\text { Bestellinformationen in das digitale } \\
\text { System eintragen }\end{array}$ & 0 & 3 & 1 & & & & \\
\hline $\begin{array}{l}\text { Umgang mit digitalen Daten: } \\
\text { systematisches Evaluieren digitaler } \\
\text { Einkaufsdaten }\end{array}$ & 0 & 3 & 1 & & & & \\
\hline
\end{tabular}

Soll I Gegenwartsbezogene Soll-Ausprägungsgrade, Soll II Zukunftsbezogene Soll-Ausprägungsgrade, Ist Aktuelle Kompetenzgrade der/des Mitarbeitenden 
Kompetenzprofil abgebildeten zukunftsbezogenen SollKompetenzgrade mit den Ist-Kompetenzgraden der jeweiligen Mitarbeitenden verglichen (vgl. Abschn. 4.2.2). Es wird deutlich, dass der Einkäufer im zukünftigen Bestandsmanagementprozess zur Ermittlung des Bedarfs an Glasware (Prozessschritt 1) für das Erzeugen digitaler Daten und den Umgang mit digitalen Daten vertiefte Kenntnisse und einen sicheren Beherrschungsgrad auch in abweichenden Arbeitssituationen benötigt. Da er jedoch nur über Grundkenntnisse bzw. einen grundlegenden Beherrschungsgrad verfügt, entstehen hier Entwicklungsbedarfe (vgl. Tab. 8). Demgegenüber verfügt er bei beiden Prozessschritten über den erforderlichen Kompetenzgrad für das ganzheitliche Denken. Weiterhin wird deutlich, dass an die Kompetenzen Anwendung von Fachwissen und Anwendung von Erfahrungswissen bei Prozessschritt 1 zukünftig geringere Anforderungen gestellt werden und daher sogar eine Überqualifizierung des Einkäufers entsteht. Bei der Planung von Einkäufen (Prozessschritt 2) wird der Einkäufer zukünftig sowohl im Hinblick auf das geforderte Fachwissen als auch in Bezug auf die Kommunikationsfähigkeit überqualifiziert sein.

Um darüber hinaus die Entwicklungsbedarfe einzelner Tätigkeitsbereiche zu erfassen, werden die im zukünftigen, digitalisierten Prozess geforderten Soll-Kompetenzgrade mit den im aktuellen Prozess benötigten Soll-Kompetenzgraden verglichen. Aus dem zukunfts- und prozessbezogenen Kompetenzprofil des Einkäufers geht hervor, dass die Anforderungen an die Anwendung von Fachwissen bei Prozessschritt 1 und 2 geringer werden: Während im aktuellen Prozess bei beiden Prozessschritten noch ein Ausprägungsgrad von 4 notwendig ist, wird im zukünftigen Prozess bei Prozessschritt 1 nur noch ein Ausprägungsgrad von 2 und bei Prozessschritt 2 ein Ausprägungsgrad von 3 gefordert (vgl. Tab. 8). Dies hängt damit zusammen, dass das digitale System alle produktrelevanten Informationen bereitstellt. Auch die Anforderungen an das Erfahrungswissen sinken bei Prozessschritt 1. Dies lässt sich auf die Tatsache zurückführen, dass zukünftig die Erfahrungswerte aller Mitarbeitenden im System hinterlegt sind und bei Bedarf eingesehen werden können. Die Anforderungen an das ganzheitliche Denken verändern sich demgegenüber nicht im zukünftigen Prozess. Hier ist weiterhin ein Kompetenzgrad von 3 notwendig. Da das digitale System zwar die Informationsweitergabe erleichtert, jedoch die persönliche Kommunikation der Abteilungen untereinander nicht vollständig ersetzt, ist für die Kommunikationsfähigkeit bei Prozessschritt 2 noch ein Kompetenzgrad von 2 notwendig. Abschließend wird deutlich, dass die Anforderungen an die digitale Kompetenz bei Prozessschritt 1 am stärksten wachsen: Während im aktuellen Prozess noch keine digitalen Kompetenzen benötigt werden, wird im zukünftigen Prozess sowohl für das Erzeugen digitaler Daten als auch für den Umgang mit digitalen Daten ein Kompetenzgrad von 3 gefordert.

\subsubsection{Kompetenzentwicklungsbedarf auf Ebene bestimmter Mitarbeitendengruppen}

Um die Kompetenzentwicklungsbedarfe bestimmter Mitarbeitendengruppen zu identifizieren, kann anhand der prozessbezogenen Kompetenzprofile verglichen werden, wie oft dieselben Kompetenzen im aktuellen und im zukünftigen, digitalisierten Prozess erforderlich sind. Werden bestimmte Kompetenzen im digitalisierten Prozess (abgebildet in den zukunfts- und prozessbezogenen Kompetenzprofilen) viel häufiger als im aktuellen Prozess (abgebildet in den gegenwarts- und prozessbezogenen Kompetenzprofilen) gefordert, entstehen Entwicklungsbedarfe. Abb. 2 stellt dar, in wie viel Prozent aller Prozessschritte des gegenwärtigen und zukünftigen Bestandsmanagementprozesses von KMU 1 die einzelnen Kompetenzen erforderlich sind bzw. sein werden und vergleicht dies mit den Ergebnissen aus KMU 2 und 3. Die Mitarbeitendengruppen, die hier im Fokus stehen, sind alle Beschäftigten, die an den zu digitalisierenden Arbeits- bzw. Geschäftsprozessen der $3 \mathrm{KMU}$ beteiligt sind. Zur Aggregation der Daten von KMU 2 und 3 wurden die in den prozessbezogenen Kompetenzprofilen aufgeführten Kompetenzen der Beschäftigten summiert und gemittelt.

Es wird deutlich, dass Fachkompetenzen zwar teilweise an Bedeutung verlieren, jedoch insgesamt noch immer sehr wichtig bleiben. Insbesondere das Fachwissen ist weiterhin die am meisten geforderte Kompetenz in allen drei KMU. Der ebenfalls erkennbare Rückgang bei den Fertigkeiten, welcher in KMU 2 und 3 noch größer ist als in KMU 1, ist auf die Tatsache zurückzuführen, dass einige manuelle Tätigkeiten in den KMU zukünftig von digitalen Systemen übernommen werden. Aufgrund der zunehmenden Komplexität und Vernetzung der digitalisierten Prozesse, gewinnt die Methodenkompetenz in KMU 1 stark an Bedeutung. Dies trifft bspw. auf die Fähigkeiten zur Problemlösung, Reflexion und zum ganzheitlichen Denken zu. Demgegenüber verlieren in KMU 2 und 3 die Reflexion und das ganzheitliche Denken sogar an Bedeutung und auch die Planungsfähigkeit wird hier nur marginal wichtiger. Dies hängt damit zusammen, dass die Prozesse durch den Einsatz digitaler Technologien verschlankt wurden und folglich weniger kognitive Aktivitäten gefordert werden. In allen drei KMU ist der Bedeutungszuwachs der digitalen Kompetenzen sehr hoch. Beispielsweise werden hier die Fähigkeiten zum Erzeugen digitaler Daten sowie im Umgang mit digitalen Daten relevant. Im Zuge der steigenden Vernetzung einzelner Geschäftsbereiche und -prozesse gewinnen darüber hinaus die Sozialkompetenzen der Mitarbeitenden an Bedeutung. Wichtig werden in KMU 1 bspw. Team- und 
Abb. 2 Kompetenzentwicklungsbedarfe der drei KMU Fig. 2 Competence development needs of the three SMEs

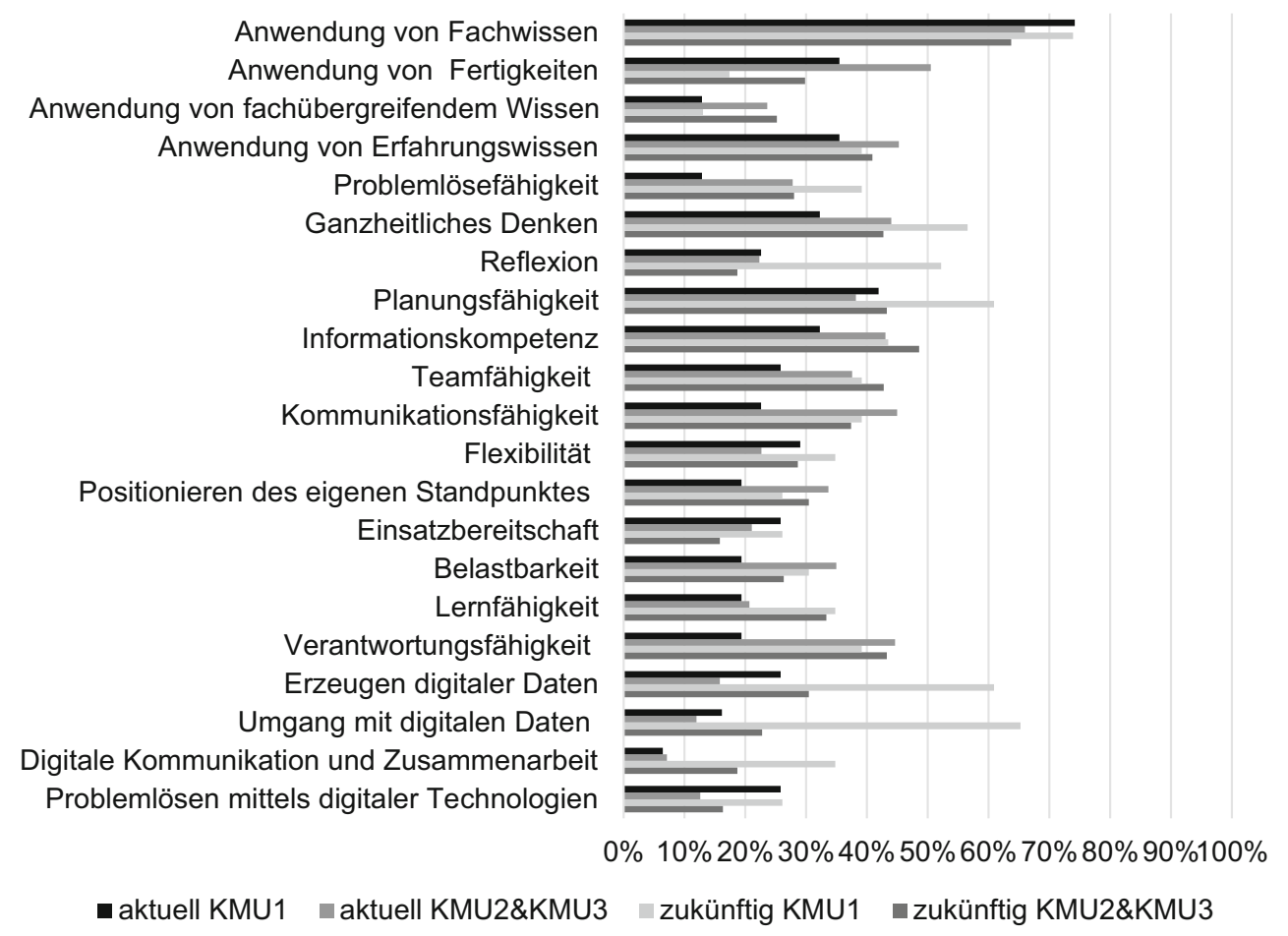

Anwendung von Fachwissen Anwendung von fachübergreifendem Wissen Anwendung von Erfahrungswissen Problemlösefähigkeit Denken

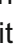

\section{.}

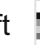

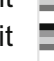

zukünftig KMU1 zukünftig KMU2\&KMU3
Kommunikationsfähigkeiten sowie die Flexibilität der Mitarbeitenden. Während die Teamfähigkeit und Flexibilität auch in KMU 2 und 3 wichtiger werden, sinkt hier jedoch die Relevanz der Kommunikationsfähigkeit. Dies hängt damit zusammen, dass in KMU 2 und 3 neue Kommunikationstechnologien eingeführt werden, welche die direkte Interaktion zwischen den Mitarbeitenden maßgeblich unterstützen. Da die Mitarbeitenden der drei KMU den Umgang mit den neuen digitalen Technologien noch erlernen müssen, wird im Hinblick auf den Bereich der Selbstkompetenz insbesondere die Lernfähigkeit wichtig. Aufgrund der hohen Informationsmengen, die durch das digitale System bereitgestellt werden, gewinnen in KMU 1 die Belastbarkeit der Mitarbeitenden sowie ihre Fähigkeit, Verantwortung zu übernehmen, stark an Bedeutung. Im Gegensatz dazu verliert die Belastbarkeit in KMU 2 und 3 an Relevanz, was mit der Tatsache zusammenhängt, dass die digitalen Technologien in der Zukunft bestimmte Aufgaben und Tätigkeiten der Mitarbeitenden übernehmen werden.

\section{Diskussion}

Das präsentierte Vorgehen ermöglicht die prospektive und prozessbezogene Erfassung und Analyse der im Digitalisierungskontext zukünftig geforderten Kompetenzen. Nachfolgend werden nochmals die zentralen Ergebnisse präsentiert sowie bestehende Limitationen und Forschungsbedarfe erläutert. Der Beitrag schließt mit der Formulierung wissenschaftlicher und praktischer Implikationen.

\subsection{Zentrale Ergebnisse}

Das entwickelte Instrumentarium kombiniert, angelehnt an die TAToo (Koch und Westhoff 2012), verschiedene Verfahren der Anforderungsanalyse (z. B. WDQ, Stegmann et al. 2010; CIT, Flanagan 1954) und umfasst einen Interviewleitfaden sowie zwei integrierte Begleitinstrumente (vgl. Abschn. 3.1.1). Durch den prozessbezogenen Einsatz des Instrumentariums lassen sich prozessbezogene Kompetenzprofile entwickeln, um darauf aufbauend zukünftige Kompetenzentwicklungsbedarfe prospektiv abzuleiten. Soll der Entwicklungsbedarf auf Ebene einzelner Mitarbeitender erfasst werden, erfolgt eine Soll-Ist-Analyse (vgl. Abschn. 4.2.3). Die im Beitrag beispielhaft vorgenommene Soll-Ist-Analyse für den Einkäufer (KMU 1) verdeutlichte, dass zur Ermittlung des Bedarfs an Glasware im zukünftigen Prozess vertiefte Kenntnisse im Hinblick auf das Erzeugen digitaler Daten und den Umgang mit digitalen Daten erforderlich sind. Da der Einkäufer jedoch nur über Grundkenntnisse verfügt, werden an dieser Stelle Entwicklungsbedarfe entstehen. Um zukünftige Entwicklungsbedarfe auf Ebene einzelner Tätigkeitsbereiche herzuleiten, werden die im aktuellen Prozess geforderten Soll-Kompetenzen mit den im zukünftigen, digitalisierten Prozess geforderten Soll-Kompetenzen verglichen. Auch hier zeigte sich, dass die Anforderungen an die digitale Kompetenz für die Position des Einkaufs am meisten steigen werden. Demgegenüber werden die Anforderungen an die Anwendung von Fachwissen sinken, da erforderliche Informationen im digitalen Bestandsmanagementsystem 
jederzeit einsehbar sind. Darüber hinaus wurde eine auf Häufigkeitseinstufungen basierende Auswertung der prozessbezogenen Kompetenzprofile der drei KMU durchgeführt, um die Entwicklungsbedarfe für die Gesamtgruppe aller am Bestandsmanagementprozess von KMU 1 beteiligten Mitarbeitenden zu erfassen und mit den Ergebnissen der anderen beiden KMU zu vergleichen. Übereinstimmend mit dem aktuellen Forschungsstand (z. B. Carretero et al. 2017; Finegold und Notabartolo 2010) zeigen die Ergebnisse, dass im Zuge der digitalen Transformation bspw. Fähigkeiten im Umgang mit digitalen Daten und Systemen, Teamfähigkeiten sowie die Flexibilität der Mitarbeitenden an Bedeutung gewinnen (vgl. Abb. 2).

Das präsentierte Vorgehen berücksichtigt vielfältige Gütekriterien. Um eine ausreichende Durchführungsobjektivität, d.h. eine von der/dem InterviewerIn weitestgehend unabhängige Datensammlung (Moosbrugger und Kelava 2008) zu gewährleisten, erfolgte die Kompetenzerfassung unter Einsatz standardisierter Befragungs- und Begleitinstrumente. Zudem wurden die ExpertInnen vor und während der Interviews sowohl mündlich als auch schriftlich über die Interviewinhalte und -abläufe informiert. Darüber hinaus wird die Auswertungsobjektivität, d.h. die Unabhängigkeit der Datenauswertung von der auswertenden Person (Moosbrugger und Kelava 2008), berücksichtigt, indem in beiden Analysephasen die Interrater-Reliabilität berechnet wurde. Die jeweils erzielten Cohens-Kappa-Werte deuteten gemäß Altman (1991) darauf hin, dass beide BeurteilerInnen die Textstellen mit einer guten Übereinstimmung in das Kategoriensystem eingeordnet haben (vgl. Abschn. 3.1.4 und 4.1.4). Zuletzt adressiert das präsentierte Vorgehen das Kriterium der Validität, welches angibt, wie zutreffend ein Instrument einen bestimmten Sachverhalt misst (Koch 2010; Moosbrugger und Kelava 2008). Um eine valide Kompetenzerfassung zu ermöglichen, wird im Rahmen des präsentierten Vorgehens ein multimethodales Instrumentarium eingesetzt. Die Kompetenzen lassen sich auf diese Weise umfassend und multiperspektivistisch ermitteln. Zur Validitätsprüfung der entwickelten Kompetenzprofile erfolgte abschließend eine kommunikative Validierung durch die befragten ExpertInnen (vgl. Abschn. 3.1.6 und 4.1.6).

\subsection{Limitationen und zukünftige Forschung}

Da das Vorgehen im KMU-Kontext erprobt wurde, ist seine Anwendbarkeit auf größere Unternehmen eingeschränkt. Zukünftige Forschung sollte das präsentierte Vorgehen daher auch in größeren Unternehmen erproben und weiterentwickeln. Um möglichst umfassende und aussagekräftige Ergebnisse zu erzielen, sollten darüber hinaus die Größe und Diversität der Stichprobe erhöht werden. Eine weitere Limitation besteht darin, dass das Vorgehen sehr zeitaufwändig ist und daher nicht alle im WDQ (Stegmann et al. 2010) aufgeführten Dimensionen zur Erfassung der Arbeitsgestaltungsmerkmale berücksichtigt. Um die Dauer der Interviews angemessen zu gestalten, sollten die Interviewten bereits vor Interviewbeginn mit den Inhalten des Fragebogens und der Begleitinstrumente vertraut gemacht werden. $\mathrm{Zu}$ dem könnten zukünftige Forschungsarbeiten eine Kurzfassung des präsentierten Vorgehens entwickeln, in der je nach Anwendungskontext nur bestimmte Fragestellungen bzw. Themenbereiche fokussiert werden. Eine weitere Limitation liegt darin, dass die Genauigkeit der erfassten Kompetenzen von dem Abstraktionsgrad der in den Interviews eingesetzten Prozessgraphik abhängt. Im Hinblick auf die Validität der prospektiven Kompetenzanalysen gilt es darüber hinaus zu evaluieren, ob die durch den präsentierten Ansatz ermittelten prospektiven Kompetenzanforderungen tatsächlich in den digital neugestalteten Prozessen relevant wurden. Da in diesem Beitrag bereits die praktische Durchführbarkeit des präsentierten Vorgehens bestätigt werden konnte, bietet es sich für zukünftige Forschungsarbeiten an, die wissenschaftlich fundierte Entwicklung dieses Vorgehens zu beschreiben. Interessant wäre schließlich zu erproben, ob und wie das Vorgehen darüber hinaus durch weitere Erhebungsinstrumente und -methoden ergänzt werden könnte. In der aktuellen Personalmanagement-Forschung gewinnen bspw. digitale Analyseinstrumente wie VR- und AR-Brillen zur Darstellung veränderter bzw. neuer Prozesse (z.B. Khandelwal und Upadhyay 2021; Sorko und Brunnhofer 2019) an Bedeutung. Die Erprobung des präsentierten Vorgehens in einer digitalen Umgebung bietet neue Erkenntnisse für die Weiterentwicklung des Konzeptes zur prospektiven und prozessbezogenen Kompetenzanalyse.

\subsection{Implikationen}

Zentrale Elemente des präsentierten Vorgehens sind die im Interviewleitfaden integrierten Begleitinstrumente. Dabei handelt es sich zum einen um den auf einer angepassten Version des WDQ (Stegmann et al. 2010) basierenden Einschätzungsbogen zu den Arbeitsgestaltungsmerkmalen und zum anderen um das auf verschiedenen konzeptionellen Ansätzen (Kompetenzatlas, Heyse und Erpenbeck 2009; KRI, Kauffeld 2010; DigComp 2.1, Carretero et al. 2017) beruhende Kompetenzinventar. Da die Begleitinstrumente unter Bezugnahme auf einen bestimmten Arbeitsbzw. Geschäftsprozess angewendet werden, lassen sich zukünftige Anforderungen und Kompetenzen im Sinne der arbeitsplatzanalytisch-empirischen Methode sehr präzise und umfassend ableiten. Nicht nur die hier vorgenommene Verknüpfung von prozess- und zukunftsbezogenen Analyseverfahren, sondern auch die Integration bestehender arbeitsund anforderungsanalytischer Methoden tragen zur Weiter- 
entwicklung anforderungsanalytischer Ansätze im Rahmen des Kompetenzmanagements bei.

Das präsentierte Vorgehen bietet Unternehmen die Möglichkeit, prozessbezogene Kompetenzprofile zu erstellen, um zukünftige Entwicklungsbedarfe prospektiv und prozessbezogen abzuleiten. Darauf basierend lassen sich Kompetenzentwicklungsmaßnahmen in Bezug auf bestimmte Arbeits- bzw. Geschäftsprozesse konzipieren. Durch den Einsatz des WDQ (Stegmann et al. 2010) ist es zudem möglich, systematisch Informationen zur prospektiven Arbeitsgestaltung im digitalisierten Geschäftsprozess zu erhalten und darauf aufbauend die Arbeitsumgebung zu optimieren und lernförderlich zu gestalten (Schaper 2019). Eine solche individuelle und zukunftsgerichtete Kompetenzentwicklung der Mitarbeitenden ist eine wichtige und notwendige Voraussetzung, um im digitalen Wandel wettbewerbsfähig zu bleiben.

Förderung Diese Studie wurde im Rahmen des Forschungsprojektes SoDigital ,Sozio-digitale Innovation durch partizipative Prozessgestaltung im virtuellen Raum" durchgeführt und durch das Bundesministerium für Bildung und Forschung (FKZ: 02L18B570, kofinanziert durch den Europäischen Sozialfonds) gefördert.

Funding Open Access funding enabled and organized by Projekt DEAL.

Open Access Dieser Artikel wird unter der Creative Commons Namensnennung 4.0 International Lizenz veröffentlicht, welche die Nutzung, Vervielfältigung, Bearbeitung, Verbreitung und Wiedergabe in jeglichem Medium und Format erlaubt, sofern Sie den/die ursprünglichen Autor(en) und die Quelle ordnungsgemäß nennen, einen Link zur Creative Commons Lizenz beifügen und angeben, ob Änderungen vorgenommen wurden.

Die in diesem Artikel enthaltenen Bilder und sonstiges Drittmaterial unterliegen ebenfalls der genannten Creative Commons Lizenz, sofern sich aus der Abbildungslegende nichts anderes ergibt. Sofern das betreffende Material nicht unter der genannten Creative Commons Lizenz steht und die betreffende Handlung nicht nach gesetzlichen Vorschriften erlaubt ist, ist für die oben aufgeführten Weiterverwendungen des Materials die Einwilligung des jeweiligen Rechteinhabers einzuholen.

Weitere Details zur Lizenz entnehmen Sie bitte der Lizenzinformation auf http://creativecommons.org/licenses/by/4.0/deed.de.

\section{Literatur}

Altman DG (1991) Practical statistics for medical research. Chapman \& Hall, London

Blickle G (2019) Anforderungsanalyse. In: Nerdinger FW, Blickle G, Schaper N (Hrsg) Arbeits- und Organisationspsychologie, 4. Aufl. Springer, Berlin, S 236-249 https://doi.org/10.1007/978-3-66256666-4_15

Blumberg VSL, Kauffeld S (2021) Kompetenzen und Wege der Kompetenzentwicklung in der Industrie 4.0. Gruppe Interakt Organ 52:203-225. https://doi.org/10.1007/s11612-021-00579-5

Campion MA, Fink AA, Ruggeberg BJ, Carr L, Phillips GM, Odman RB (2011) Doing competencies well: best practices in competency modeling. Pers Psychol 64:225-262

Carretero S, Vuorikari R, Punie Y (2017) DigComp 2.1: the digital competence framework for citizens with eight proficiency levels and examples of use. Publications Office of the European Union, Luxembourg, S 1-48 https://doi.org/10.2760/38842

Carstensen T (2015) Neue Anforderungen und Belastungen durch digitale und mobile Technologien. WSI Mitt 3(2015):187-193

Cohen J (1960) A coefficient of agreement for nominal scales. Educ Psychol Meas 20:37-46

Coovert MD, Thompson LF (2014) Toward a synergistic relationship between psychology and technology. In: Coovert MD, Thompson L (Hrsg) The psychology of workplace technology. Routledge, New York

Decius J, Schaper N (2017) The competence management tool (CMT) - a new instrument to manage competences in small and medium-sized manufacturing enterprises. Proc Manuf 9:376-383. https://doi.org/10.1016/j.promfg.2017.04.041

Dengler K, Matthes B (2015) Folgen der Digitalisierung für die Arbeitswelt. Substituierbarkeitspotenziale von Berufen in Deutschland. IAB Forschungsbericht 11/2015

Eck CD, Rietiker J (2010) Kompetenzen und Anforderungsanalyse. In: Werkmann-Karcher B, Rietiker J (Hrsg) Angewandte Psychologie für das Human Resource Management. Konzepte und Instrumente für ein wirkungsvolles Personalmanagement. Springer, Berlin Heidelberg, S 180-214 https://doi.org/10.1007/978-3-64212481-5_10

Eck CD, Jöri H, Vogt M (2007) Assessment-Center, 2. Aufl. Springer, Heidelberg

Eckhardt HH, Schuler H (1992) Berufseignungsdiagnostik. In: Jäger RS, Petermann F (Hrsg) Psychologische Diagnostik, 2. Aufl. Psychologie Verlags Union, Weinheim, S 533-551

Enke J, Glass R, Kreß A, Hambach J, Tisch M, Metternich J (2018) Industrie 4.0-Competencies for a modern production system. A curriculum for learning factories. Proc Manuf 23(2018):267-272

Ferrari A (2012) Digital competence in practice: an analysis of frameworks. European Comission, Joint Research Centre, Institute for Prospective Technological Studies, Seville, S 1-91 https://doi. org/10.2791/82116

Ferrari A (2013) DigComp: a framework for developing and understanding digital competence in Europe. European Comission, Joint Research Centre, Institute for Prospective Technological Studies, Seville, S 1-46 https://doi.org/10.2788/52966

Finegold D, Notabartolo AS (2010) 21st century competencies and their impact: an interdisciplinary literature review. In: Finegold D, Gatta M, Salzman H, Schurman SJ (Hrsg) Transforming the US workforce development system. Labor and Employment Relations Association, Illinois, S 19-56

Flanagan JC (1954) The critical incident technique. Psychol Bull 51(4):327-358

Flick U (2020) Gütekriterien qualitativer Forschung. In: Mey G, Mruck K (Hrsg) Handbuch qualitative Forschung in der Psychologie. Springer, Wiesbaden, S 247-263 https://doi.org/10.1007/ 978-3-658-26887-9_30

Galiläer L (2006) Segmentierung von Anforderungsniveaus - Das Erkenntnispotenzial von Qualifikationsentwicklungsforschung. Berufs- und Wirtschafspädagogik - online (bwpat) 11:1-16. http:// www.bwpat.de/ausgabe11/galilaeer_bwpat11.pdf. Zugegriffen: 12. Apr. 2021

Gessler M, Sebe-Opfermann A (2016) Kompetenzmodelle. In: MüllerVorbrüggen M, Radel J (Hrsg) Handbuch Personalentwicklung: Die Praxis der Personalbindung, Personalförderung und Arbeitsstrukturierung, 4. Aufl. Schäffer-Poeschel, Stuttgart, S 159-184

Grote S, Kauffeld S, Frieling E (2006) Vom Wettbewerb zur Kompetenz. In: Grote S, Kauffeld S, Frieling E (Hrsg) Kompetenzmanagement - Grundlagen und Praxisbeispiele. Schäffer-Poeschel, Stuttgart, S 1-14

Hamborg K-C, Schaper N (2018) Analyse, Bewertung und Gestaltung von Aufgaben, Arbeitstätigkeiten und Arbeitssystemen. In: Greif S, Hamborg K-C (Hrsg) Methoden der Arbeits-, Organisations- und Wirtschaftspsychologie. Enzyklopädie der Psychologie, Bd. B/III/3. Hogrefe, Göttingen, S 1-79 
Harteis C (2018) Machines, change and work: an educational view on the digitalization of work. In: Harteis C (Hrsg) The impact of digitalization in the workplace: an educational view. Springer, Cham, S 1-12 https://doi.org/10.1007/978-3-319-63257-5_1

Hasenau K, Sende C, Nick C (2013) Erstellung prozessbezogener Kompetenzmatrizen in produzierenden KMU. In Schlick CM (Hrsg) Handreichungen für die betriebliche Praxis. Institut für Arbeitswissenschaft (IAW) der RWTH Aachen, Aachen, S 1-8

Hegmanns T, Straub N, Kaczmarek S, Rudolph B, Sobiech D, Müller S, Dehler J, Haertel T, May D, Radtke M, Neubauer D, Möllmann A, Zaremba B (2019) Kompetenzmanagement in der Logistik der Zukunft - ein Umsetzungsbeispiel von der Modellierung und Diagnostik zur unternehmensspezifischen und individuellen Kompetenzentwicklung. In: Bullinger-Hoffmann AC (Hrsg) Zukunftstechnologien und Kompetenzbedarfe: Kompetenzentwicklung in der Arbeitswelt 4.0. Springer, Berlin, S 199-232 https:// doi.org/10.1007/978-3-662-54952-0_12

Heyse V (2010) Verfahren zur Kompetenzermittlung und Kompetenzentwicklung. In: Heyse V, Erpenbeck J, Ortmann S (Hrsg) Grundstrukturen menschlicher Kompetenzen: Praxiserprobte Konzepte und Kompetenzen, Bd. 5. Waxmann, Münster, S 55-174

Heyse V, Erpenbeck J (2009) Kompetenztraining: Informations- und Trainingsprogramme, 2. Aufl. Schäffer-Poeschel, Stuttgart

Hirsch-Kreinsen H (2017) Digitalisierung industrieller Einfacharbeit: Entwicklungspfade und arbeitspolitische Konsequenzen. Arbeit 26(1):1-26

Höft S, Goerke P (2014) Traditionelle Arbeits- und Anforderungsanalyse trifft modernen Kompetenzmanagementansatz: Rosenkrieg oder Traumhochzeit? Wirtschaftspsychologie 16(1):5-14

Kato-Beiderwieden A-L, Schlicher KD, Ötting SK, Heppner H, Maier GW (2021) Prospektive Kompetenzanalyse (ProKA) - Ein Verfahren zur Einschätzung von zukünftigen Kompetenzveränderungen. Gruppe Interakt Organ 52:245-260. https://doi.org/10.1007/ s11612-021-00577-7

Kauffeld S (2010) KRI - Kompetenz-Reflexions-Inventar. In: Sarges W, Wottaw H, Roos C (Hrsg) Organisationspsychologische Instrumente. Handbuch wirtschaftlicher Testverfahren, Bd. II. Pabst Science Publishers, Lengerich, S 201-208

Kauffeld S, Grote S (2019) Personalentwicklung. In: Kauffeld S (Hrsg) Arbeits-, Organisations- und Personalpsychologie für Bachelor, 3. Aufl. Springer, Berlin, S 167-210 https://doi.org/10.1007/9783-662-56013-6

Khandelwal K, Upadhyay AK (2021) Virtual reality interventions in developing and managing human resources. Hum Res Dev Int 24(2):219-233. https://doi.org/10.1080/13678868.2019.1569920

Kinkel S, Schemmann B, Lichtner R (2017) Critical competencies for the innovativeness of value creation champions: identifying challenges and work-integrated solutions. Proc Manuf 9:323-330. https://doi.org/10.1016/j.promfg.2017.04.021

Koch A (2010) Die Task-Analysis-Tools (TAToo) - Entwicklung, empirische und praktische Prüfungen eines Instrumentes für Anforderungsanalysen. Dissertationsschrift. Fakultät Mathematik und Naturwissenschaften der Technischen Universität Dresden, Dresden

Koch A, Westhoff K (2012) Task-Analysis Tools (TAToo) - Schritt für Schritt Unterstützung zur erfolgreichen Anforderungsanalyse. Pabst Science Publishers, Lengerich

Koch A, Strobel A, Miller R, Garten A, Cimander C, Westhoff K (2012) Never use one when two will do-the effects of a multiperspective approach on the outcome of job analyses using the critical incident technique. J Pers Psychol 11(2):95-102. https:// doi.org/10.1027/1866-5888/a000060

Krüger D, Riemeier T (2014) Die qualitative Inhaltsanalyse - eine Methode zur Auswertung von Interviews. In: Krüger D, Parchmann I, Schecker H (Hrsg) Methoden in der naturwissenschaftsdidaktischen Forschung. Springer, Berlin Heidelberg, S 133-145 https:// doi.org/10.1007/978-3-642-37827-0_11
Krüger C, Utte A, Rowold J (2015) Arbeits- und Anforderungsanalyse. In: Rowold J (Hrsg) Human Resource Management. Lehrbuch für Bachelor und Master, 2. Aufl. Springer Gabler, Berlin Heidelberg, S 59-71 https://doi.org/10.1007/978-3-662-45983-6_7

Krumm S, Mertin I, Dries C (2012) Kompetenzmodelle. Hogrefe, Göttingen

Van Laar E, van Deursen AJAM, van Dijk JAGM, de Haan J (2017) The relation between 21 st-century skills and digital skills: a systematic literature review. Comput Human Behav 72:577-588. https://doi. org/10.1016/j.chb.2017.03.010

Van Laar E, van Deursen AJAM, van Dijk JAGM, de Haan J (2020) Determinants of 21 st-century skills and 21 st-century digital skills for workers: a systematic literature review. Sage Open. https://doi. org/10.1177/2158244019900176

Lebrenz C (2020) Strategie und Personalmanagement. Konzepte und Instrumente zur Umsetzung im Unternehmen, 2. Aufl. Springer Gabler, Wiesbaden https://doi.org/10.1007/978-3-658-29033-7

Leyh C, Bley K (2016) Digitalisierung: Chance oder Risiko für den deutschen Mittelstand? - Eine Studie ausgewählter Unternehmen. HMD 53:29-41. https://doi.org/10.1365/s40702-015-0197-2

Lorenz M, Rohrschneider U (2015) Erfolgreiche Personalauswahl. Sicher, schnell und durchdacht, 2. Aufl. Springer Gabler, Wiesbaden https://doi.org/10.1007/978-3-8349-4766-6_1

Lozo L, Bipp T, Steinmayr R, Blümke M (2015) Anforderungsanalyse für offene Positionen in der Wissenschaft. In: Peus C, Braun S, Hentschel T, Frey D (Hrsg) Personalauswahl in der Wissenschaft. Evidenzbasierte Methoden und Impulse für die Praxis. Springer, Berlin Heidelberg, S 51-64 https://doi.org/10.1007/978-3-66248112-7_4

Mayring P (2010) Qualitative Inhaltsanalyse. In: Mey G, Mruck K (Hrsg) Handbuch Qualitative Forschung in der Psychologie, 1. Aufl. VS, Wiesbaden, S 601-613

Moosbrugger H, Kelava A (2008) Qualitätsanforderungen an einen psychologischen Test (Testgütekriterien). In: Moosbrugger H, Kelava A (Hrsg) Testtheorie und Fragebogenkonstruktion. Springer, Heidelberg, S 7-26

Morgeson FP, Humphrey SE (2006) The work design questionnaire (WDQ): developing and validating a comprehensive measure for assessing job design and the nature of work. J Appl Psychol 91(6):1321-1339. https://doi.org/10.1037/0021-9010.91.6.1321

Noll A (2010) Kompetenzmanagement in Unternehmen. Auf dem Weg zu einer kompetenzbasierten Personalentwicklung. Berufsbild Wiss Prax 39(20155):31-33

Okoli C (2015) A guide to conducting a standalone systematic literature review. CAIS 37(43):879-910

Paruzel A, Bentler D, Schlicher KD, Nettelstroth W, Maier GW (2020) Employees first, technology second. Implementation of smart glasses in a manufacturing company. Z Arbeits Organisationspsychol 64(1):46-57. https://doi.org/10.1026/0932-4089/a000292

Rothe I, Wischniewski S, Tegtmeier P, Tisch A (2019) Arbeiten in der digitalen Transformation - Chancen und Risiken für die menschengerechte Arbeitsgestaltung. Z Arb Wiss 73:246-251. https:// doi.org/10.1007/s41449-019-00162-1

Sauter W, Staudt F-P (2016) Strategisches Kompetenzmanagement 2.0. Potenziale nutzen - Performance steigern. Springer Gabler, Wiesbaden https://doi.org/10.1007/978-3-658-11294-3_1

Schaper N (2019) Arbeitsanalyse und -bewertung. In: Nerdinger FW, Blickle G, Schaper N (Hrsg) Arbeits- und Organisationspsychologie, 4. Aufl. Springer, Berlin, S 385-410 https://doi.org/10.1007/ 978-3-662-56666-4_21

Schuler H (2002) Das Einstellungsinterview. Hogrefe, Göttingen

Schuler H (2014) Arbeits- und Anforderungsanalyse. In Schuler H, Kanning UP (Hrsg) Lehrbuch der Personalpsychologie, Bd. 3. Hogrefe, Göttingen, S 61-98

Sonntag K, Schaper N (1999) Förderung beruflicher Handlungskompetenz. In: Sonntag K (Hrsg) Personalentwicklung in Organisationen. Hogrefe, Göttingen, S 211-244 
Sonntag K, Schaper N (2016) Berufliche Handlungskompetenz fördern: Wissens- und verhaltensbasierte Verfahren. In: Sonntag K (Hrsg) Personalentwicklung in Organisationen: Psychologische Grundlagen, Methoden und Strategien, 4. Aufl. Hogrefe, Göttingen, S 61-97

Sonntag K, Schmidt-Rathjens C (2005) Anforderungsanalyse und Kompetenzmodelle. In: Gonon P, Klauser F, Nickolaus R, Huisinga R (Hrsg) Kompetenz, Kognition und neue Konzepte der beruflichen Bildung, 1. Aufl. VS, Wiesbaden, S 55-66

Sonntag K, Stegmaier R (2005) Lernkulturen verstehen, gestalten und messen. Das Lernkulturinventar als organisationsdiagnostisches Verfahren zur Messung von Lernkultur. Personalführung 1:22-29

Sorko SR, Brunnhofer M (2019) Potentials of augmented reality in training. Proc Manuf 31:85-90

Sprafke N, Hohagen S, Erlinghagen M, Nolte A, Wenig P, Zechmann A, Wilkens U, Minssen H, Herrmann TA (2019) Voraussetzungen der erfolgreichen Implementierung von Kompetenzmanagement in KMU. In: Bullinger-Hoffmann AC (Hrsg) Zukunftstechnologien und Kompetenzbedarfe. Kompetenzentwicklung in der Arbeitswelt 4.0. Springer, Berlin, S 62-82 https://doi.org/10. 1007/978-3-662-54952-0_5

Stegmann S, van Dick R, Ullrich J, Charalambous J, Menzel B, Egold N, Wu T-CT (2010) Der Work Design Questionnaire. Vorstellung und erste Validierung einer deutschen Version. Z Arbeits Organisationspsychol 54(1):1-28. https://doi.org/10.1026/09424089/a000002

Urbach N, Ahlemann F (2019) IT management in the digital age. A roadmap for the IT department of the future. Springer, Cham https://doi.org/10.1007/978-3-319-96187-3
Urbach N, Ahlemann F, Böhmann T, Drews P, Brenner W, Schaudel F, Schütte R (2019) The impact of digitalization on the IT department. Bus Inf Syst Eng 61(1):123-131. https://doi.org/10.1007/ s12599-018-0570-0

Vernim S, Korder S, Tropschuh B (2019) Sind unsere Mitarbeiter für einen Einsatz in der digitalen Fabrik richtig qualifiziert? Ermittlung zukünftiger Mitarbeiteranforderungen in der Smart Factory. In: Bosse CK, Zink KJ (Hrsg) Arbeit 4.0 im Mittelstand. Chancen und Herausforderungen des digitalen Wandels für KMU. Springer Gabler, Berlin, S 71-90 https://doi.org/10.1007/978-3-66259474-2_5

Vom Brocke J, Simons A, Niehaves B, Riemer K, Plattfaut R, Cleven A (2009) Reconstructing the giant: on the importance of rigour in documenting the literature search process. ECIS 2009 Proceedings.

Vuorikari R, Punie Y, Carretero S, Van den Brande L (2016) DigComp 2.0: the digital competence framework for citizens. Update phase 1: the conceptual reference model. Publication Office of the European Union, Luxembourg https://doi.org/10.2791/11517

Wienkamp H (2020) Der Weg zum Personalkennzahlensystem. Das HR-Cockpit der Praxis - einfach, pragmatisch, systematisch. Springer, Berlin https://doi.org/10.1007/978-3-662-60903-3_8

Zinke G (2019) Berufsbildung 4.0 - Fachkräftequalifikationen und Kompetenzen für die digitalisierte Arbeit von morgen: Branchen- und Berufescreening: vergleichende Gesamtstudie. Wissenschaftliche Diskussionspapiere, Heft 213. Bundesinstitut für Berufsbildung (BIBB), Bonn 